JOURNAL OF THE

AMERICAN MATHEMATICAL SOCIETY

Volume 13, Number 4, Pages 697-723

S 0894-0347(00)00343-X

Article electronically published on June 22, 2000

\title{
CONVERGENCE AND FINITE DETERMINATION OF FORMAL CR MAPPINGS
}

\author{
M. S. BAOUENDI, P. EBENFELT, AND LINDA PREISS ROTHSCHILD
}

\section{$\S 0$. INTRODUCTION}

In this paper, we study the convergence and finite determination of formal holomorphic mappings of $\left(\mathbb{C}^{N}, 0\right)$ taking one real submanifold into another. By a formal (holomorphic) mapping $H:\left(\mathbb{C}^{N}, 0\right) \rightarrow\left(\mathbb{C}^{N}, 0\right)$, we mean an $N$-vector $H=\left(H_{1}, \ldots, H_{N}\right)$, where each $H_{j}$ is a formal power series in $N$ indeterminates with no constant term. If $M$ and $M^{\prime}$ are real smooth submanifolds through 0 in $\mathbb{C}^{N}$ defined near the origin by $\rho(Z, \bar{Z})=0$ and $\rho^{\prime}(Z, \bar{Z})=0$ respectively, where $\rho$ and $\rho^{\prime}$ are vector valued smooth defining functions, then we say that a formal mapping $H:\left(\mathbb{C}^{N}, 0\right) \rightarrow\left(\mathbb{C}^{N}, 0\right)$ sends $M$ into $M^{\prime}$ if the vector valued power series $\rho^{\prime}(H(Z), \overline{H(Z)})$ is a (matrix) multiple of $\rho(Z, \bar{Z})$. For real-analytic hypersurfaces, we shall prove the following.

Theorem 1. Let $M$ and $M^{\prime}$ be real-analytic hypersurfaces through the origin in $\mathbb{C}^{N}, N \geq 2$. Assume that neither $M$ nor $M^{\prime}$ contains a nontrivial holomorphic subvariety through 0 . Then any formal mapping $H:\left(\mathbb{C}^{N}, 0\right) \rightarrow\left(\mathbb{C}^{N}, 0\right)$ sending $M$ into $M^{\prime}$ is convergent.

The condition that $M^{\prime}$ above does not contain a nontrivial holomorphic subvariety is necessary (see Remark 2.3). As a corollary we obtain the following characterization.

Corollary 2. Let $M$ be a real-analytic hypersurface through 0 in $\mathbb{C}^{N}$ with $N \geq 2$. Then, $M$ does not contain a nontrivial holomorphic subvariety through 0 if and only if every formal mapping $H:\left(\mathbb{C}^{N}, 0\right) \rightarrow\left(\mathbb{C}^{N}, 0\right)$ sending $M$ into itself is convergent.

As a consequence of the geometric properties of mappings used in the proof of Theorem 1 above, we also obtain a new reflection principle for CR mappings between real-analytic hypersurfaces (see Theorem 2.4).

We state now some results for generic submanifolds of higher codimension in $\mathbb{C}^{N}$. A formal mapping $H:\left(\mathbb{C}^{N}, 0\right) \rightarrow\left(\mathbb{C}^{N}, 0\right)$ is called finite if the ideal generated by its components $H_{1}, \ldots, H_{N}$ has finite codimension in the ring of formal power series $\mathbb{C}[[Z]]$. Recall that a smooth generic submanifold $M \subset \mathbb{C}^{N}$ is of finite type (in

Received by the editors June 3, 1999.

2000 Mathematics Subject Classification. Primary 32H02.

Key words and phrases. Formal mappings, generic submanifolds, CR mappings, holomorphic mappings, finite determination.

The first and the third authors are partially supported by National Science Foundation grant DMS 98-01258. The second author is partially supported by a grant from the Swedish Natural Science Research Council. 
the sense of Kohn and Bloom-Graham) at $p \in M$ if the Lie algebra generated by its $\mathrm{CR}$ vector fields and their complex conjugates has full dimension at $p$ (see $\S 1.1$ ). For a real-analytic hypersurface in $\mathbb{C}^{2}$ the notion of finite type is equivalent to that of not containing a nontrivial variety. Our first result for higher codimensional generic submanifolds is the following.

Theorem 3. Let $M$ and $M^{\prime}$ be real-analytic generic submanifolds of the same dimension through the origin in $\mathbb{C}^{N}$. Assume that $M$ is of finite type at 0 , and that $M^{\prime}$ does not contain any germ of a nontrivial holomorphic subvariety through 0 . Then any formal finite mapping $H:\left(\mathbb{C}^{N}, 0\right) \rightarrow\left(\mathbb{C}^{N}, 0\right)$ sending $M$ into $M^{\prime}$ is convergent.

In fact, we prove a more general result (see Theorem 2.1) in which $M$ and $M^{\prime}$ need not be in the same complex space nor of the same dimension. Moreover, the condition that $M^{\prime}$ does not contain any nontrivial variety is replaced by the weaker assumption that $M^{\prime}$ is essentially finite at 0 , and the condition of finiteness of the mapping $H$ is replaced by injectivity of its Segre homomorphism. (See $\S 1.1$ for relevant definitions.)

Recall that two real submanifolds $M$ and $M^{\prime}$ of the same dimension through the origin in $\mathbb{C}^{N}$ are said to be biholomorphically equivalent at 0 if there exists a biholomorphism $H:\left(\mathbb{C}^{N}, 0\right) \rightarrow\left(\mathbb{C}^{N}, 0\right)$, defined near 0 , sending $M$ into $M^{\prime}$. Similarly, we shall say that $M$ and $M^{\prime}$ are formally equivalent (at 0 ) if there exists a formal invertible mapping sending $M$ into $M^{\prime}$.

Theorem 4. Let $M$ and $M^{\prime}$ be real-analytic generic submanifolds through the origin in $\mathbb{C}^{N}$. Assume that $M$ is of finite type at 0 and does not contain any germ of a nontrivial holomorphic subvariety through 0 . Then $M$ and $M^{\prime}$ are formally equivalent at 0 if and only if they are biholomorphically equivalent at 0.

In the following result, we use the notation $j_{p}^{k} f$ for the jet of order $k$ at $p \in M$ of a smooth mapping $f: M \rightarrow M^{\prime}$, where $M$ and $M^{\prime}$ are smooth manifolds. We shall say that a smooth generic submanifold $M$ does not contain a (nontrivial) formal variety through 0 if there is no (nontrivial) formal holomorphic curve $\gamma:(\mathbb{C}, 0) \rightarrow\left(\mathbb{C}^{N}, 0\right)$ such that $\rho(\gamma(t), \overline{\gamma(t)}) \sim 0$, where the composition is in the sense of formal power series and $\sim$ means equality for power series. Here, $\rho(Z, \bar{Z})$ is a vector valued smooth defining function for $M$ near 0 identified with its Taylor series at the origin.

Theorem 5. Let $M$ be a smooth generic submanifold through the origin in $\mathbb{C}^{N}$. Assume that $M$ is of finite type at 0 and does not contain a nontrivial formal variety through 0 . Then there exists a positive integer $K$ with the following property. If $M^{\prime}$ is a smooth generic submanifold in $\mathbb{C}^{N}$ and $H^{1}, H^{2}: M \rightarrow M^{\prime}$ are smooth local CR diffeomorphisms, defined near 0 , such that $j_{0}^{K}\left(H^{1}\right)=j_{0}^{K}\left(H^{2}\right)$, then $j_{0}^{l}\left(H^{1}\right)=$ $j_{0}^{l}\left(H^{2}\right)$ for all positive integers $l$.

Theorem 5 is a consequence of the more general result Theorem 2.5, in which $M$ and $M^{\prime}$ can be of different dimensions and be contained in different complex spaces. As in the case of Theorem 3, we may replace the condition that $M$ does not contain a nontrivial formal variety through 0 by the assumption that $M$ is essentially finite at 0 and the condition that $H^{1}, H^{2}$ are CR diffeomorphisms by the assumption that their Segre homomorphisms are injective. We should also point out that if $M$ is of D-finite type (finite type in the sense of D'Angelo [DA]) at 0 , then it does not contain a nontrivial formal variety through 0 . 
The study of formal mappings between analytic objects has a long history. We shall mention here only results which are related to this work. In their study of normal forms for real-analytic Levi nondegenerate hypersurfaces in complex space, Chern and Moser [CM] proved that any formal invertible mapping between such is convergent. We should also mention that Moser and Webster MW proved convergence of formal equivalences between real-analytic two-dimensional surfaces with complex elliptic tangents (of a certain kind) at the origin in $\mathbb{C}^{2}$. On the other hand, Moser and Webster in the same paper also proved that there are realanalytic surfaces through the origin in $\mathbb{C}^{2}$ which are formally equivalent but not biholomorphically equivalent at 0 . Subsequently, Webster [W] proved that each real Lagrangian surface in $\mathbb{C}^{2}$ with a nondegenerate complex tangent at 0 is formally equivalent to a certain quadric. Later, Gong Go2 showed that there exist such real-analytic Lagrangian surfaces which are not biholomorphic to that quadric. An analogous situation arises in the classification of glancing hypersurfaces (see Melrose [Mel, Oshima [O], and also Gong [Go1]).

Previous results on convergence of formal mappings between real-analytic submanifolds in complex space were obtained by the authors in [BER3] and [BER5], where analytic dependence of holomorphic mappings on their jets of a prescribed finite order was studied. We should point out that the results in the present paper are new even for invertible mappings. (See also the closing remark at the end of this paper.)

Finite determination (by their 2-jets) for formal invertible mappings between smooth Levi nondegenerate hypersurfaces was proved by Chern and Moser [CM] (see also earlier work of E. Cartan [C] and Tanaka [T]). Later work was done by e.g. Tumanov and Henkin [TH], Beloshapka B], and the authors [BER2, BER5]. For a more detailed history, the reader is referred to [BER5].

This paper is organized as follows. In $\S 1$, we introduce the basic concepts of formal manifolds and mappings in the setting of ideals in the ring of formal power series. We also give the fundamental properties of the Segre mappings for generic submanifolds: these mappings play an important role in the proofs. The precise formulations of our main results are given in $\S 2$. Another ingredient in the proofs is the reflection identity for formal mappings presented in $\S 3$. The proofs of the results stated above and those in $\S 2$ are then given in $\S \S 4-7$.

\section{§1. Preliminaries and Basic Definitions}

$\S 1.1$. Formal mappings and manifolds. Let $\mathbb{C}[[x]]=\mathbb{C}\left[\left[x_{1}, \ldots, x_{k}\right]\right]$ be the ring of formal power series in $x=\left(x_{1}, \ldots, x_{k}\right)$ with complex coefficients. We shall say that a proper ideal $I \subset \mathbb{C}\left[\left[x_{1}, \ldots, x_{k}\right]\right]$ is a manifold ideal of dimension $l$, if it is generated by $k-l$ power series $h_{1}, \ldots, h_{k-l}$ whose differentials at the origin are independent. This is equivalent to the local ring of $I, \mathbb{C}[[x]] / I$, being a regular local ring of Krull dimension $l$ (see e.g. [Ha], $\mathrm{AM}$ ). We shall write $\mathcal{I}\left(h_{1}(x), \ldots, h_{k-l}(x)\right)=$ $\mathcal{I}(h(x))$ for the ideal in $\mathbb{C}[x]]$ generated by $h(x)=\left(h_{1}(x), \ldots, h_{k-l}(x)\right)$.

By a formal mapping $F:\left(\mathbb{C}^{k}, 0\right) \rightarrow\left(\mathbb{C}^{m}, 0\right)$ we shall mean a vector of formal power series $F \in \mathbb{C}\left[\left[x_{1}, \ldots, x_{k}\right]\right]^{m}$ such that each component of $F=\left(F_{1}, \ldots, F_{m}\right)$ has no constant term. Writing $\mathbb{C}[[y]]=\mathbb{C}\left[\left[y_{1}, \ldots, y_{m}\right]\right]$, we let $\eta_{F}: \mathbb{C}[[y]] \rightarrow \mathbb{C}[[x]]$ be the ring homomorphism defined by

$$
\eta_{F}(g)(x)=(g \circ F)(x), \quad g \in \mathbb{C}[[y]] .
$$


Conversely, any ring holomorphism $\eta: \mathbb{C}[[y]] \rightarrow \mathbb{C}[[x]]$ is of the form (1.1.1) for some uniquely determined formal mapping $F$. Given an ideal $I \subset \mathbb{C}[[x]$, we define its pushforward by $F, F_{*}(I) \subset \mathbb{C}[[y]]$, as follows:

$$
F_{*}(I)=\{f \in \mathbb{C}[[y]]: f \circ F \in I\} .
$$

Note that $F_{*}(I)=\eta_{F}^{-1}(I)$, with $\eta_{F}$ defined by (1.1.1).

It is easy to check that $F_{*}(I)$ is an ideal and if $I$ is prime, then $F_{*}(I)$ is also prime. However, even if $I$ is a manifold ideal, $F_{*}(I)$ need not be a manifold ideal. Observe that the mapping $\eta_{F}$ is a ring isomorphism if and only if $m=k$ and the Jacobian of $F$ at 0 is invertible. In that case we shall refer to $F$ as a formal change of coordinates. If $I \subset \mathbb{C}[[x]]$ is an ideal, then $I$ is a manifold ideal of dimension $l$ if and only if there is a formal change of coordinates $F$ such that $F_{*}(I) \subset \mathbb{C}[[y]]$ is generated by the coordinates $y_{1}, \ldots, y_{k-l}$.

A formal vector field in $\mathbb{C}^{k}$ is a derivation of $\mathbb{C}[[x]]$. It is easy to check that any formal vector field $X$ can be written uniquely in the form

$$
X=\sum_{j=1}^{k} a_{j}(x) \frac{\partial}{\partial x_{j}},
$$

with $a_{j} \in \mathbb{C}[[x]]$. We shall say that $X$ is tangent to a proper ideal $I \subset \mathbb{C}[[x]]$ if for any $f \in I$ we have $X f \in I$. If $F$ is a formal change of coordinates as above, then we may define the pushforward of a formal vector field $X$ by

$$
\left(F_{*} X\right)(g)=\eta_{F^{-1}}\left(X\left(\eta_{F}(g)\right)\right), \quad g \in \mathbb{C}[[y]] .
$$

It is clear that $F_{*} X$ is also a formal vector field. Furthermore, if $X$ is tangent to an ideal $I \subset \mathbb{C}[[x]]$, then $F_{*} X$ is tangent to $F_{*}(I)$.

If $Z=\left(Z_{1}, \ldots, Z_{N}\right), \zeta=\left(\zeta_{1}, \ldots, \zeta_{N}\right)$, and $I \subset \mathbb{C}[[Z, \zeta]]$ an ideal, we shall say that $I$ is a real ideal if $f(Z, \zeta) \in I$ implies $\bar{f}(\zeta, Z) \in I$. Here $\bar{f}$ is the formal power series obtained by taking the complex conjugates of the coefficients of $f$. If $f(Z, \zeta) \in \mathbb{C}[[Z, \zeta]]$, we shall say that $f$ is real if $f(Z, \zeta)=\bar{f}(\zeta, Z)$. The notion of a real ideal is not invariant under all formal changes of coordinates in $\mathbb{C}[[Z, \zeta]$, and we shall restrict ourselves to changes of coordinates of the form $Z^{\prime}=H(Z)$, $\zeta^{\prime}=\bar{H}(\zeta)$, where $H(Z)$ is a formal change of coordinates in $\mathbb{C}^{N}$. We shall write $\mathcal{H}(Z, \zeta):=(H(Z), \bar{H}(\zeta))$. If $I$ is a real ideal in $\mathbb{C}[[Z, \zeta]]$, so is the ideal $\mathcal{H}_{*}(I)$, as defined by (1.1.2).

If $I \subset \mathbb{C}[[Z, \zeta]]$ is a real manifold ideal of dimension $2 N-d$, then one can find generators $\rho=\left(\rho_{1}, \ldots, \rho_{d}\right)$ which are real and have linearly independent differentials at the origin, i.e. satisfy

$$
\partial \rho_{1}(0) \wedge \cdots \wedge \partial \rho_{d}(0) \neq 0 .
$$

If $\rho^{\prime}=\left(\rho_{1}^{\prime}, \ldots, \rho_{d}^{\prime}\right)$ is another set of generators of $I$ (not necessarily real), then there exists a $d \times d$ matrix of formal power series $a(Z, \zeta)$ (with $a(0,0)$ necessarily invertible) such that

$$
\rho(Z, \zeta) \sim a(Z, \zeta) \rho^{\prime}(Z, \zeta)
$$

We say that a real manifold ideal $I \subset \mathbb{C}[[Z, \zeta]]$ of dimension $2 N-d$ defines a formal real submanifold $M$ of $\mathbb{C}^{N}$ through 0 of codimension $d$ (and dimension $2 N-d$ ), and we write $I=\mathcal{I}(M)$. We shall say that a formal vector field in $\mathbb{C}^{N} \times \mathbb{C}^{N}$ is tangent to $M$ if it is tangent to $\mathcal{I}(M)$. 
The motivation behind these definitions is the following. If in addition the components of the formal series $\rho$ above are convergent, then the equations $\rho(Z, \bar{Z})=0$ define a real-analytic submanifold $M$ of $\mathbb{C}^{N}$ through 0 . Also, if $M$ is a smooth real submanifold in $\mathbb{C}^{N}$ through 0 , then the Taylor series at 0 of a smooth defining function $\rho$ of $M$ near 0 defines a formal real submanifold of $\mathbb{C}^{N}$ through 0 . Thus, the notion of a formal real submanifold is a generalization of that of real-analytic and smooth real submanifolds of $\mathbb{C}^{N}$.

A formal real submanifold $M$ of $\mathbb{C}^{N}$ is said to be generic if there exist formal series $\rho$ generating $\mathcal{I}(M)$ satisfying the condition

$$
\partial_{Z} \rho_{1}(0) \wedge \cdots \wedge \partial_{Z} \rho_{d}(0) \neq 0
$$

which in particular implies (1.1.5).

If $H:\left(\mathbb{C}^{N}, 0\right) \rightarrow\left(\mathbb{C}^{N^{\prime}}, 0\right)$ is a formal mapping, then as before we associate to it a formal mapping $\mathcal{H}:\left(\mathbb{C}^{N} \times \mathbb{C}^{N}, 0\right) \rightarrow\left(\mathbb{C}^{N^{\prime}} \times \mathbb{C}^{N^{\prime}}, 0\right)$ defined by

$$
\mathcal{H}(Z, \zeta)=(H(Z), \bar{H}(\zeta))
$$

If $M$ and $M^{\prime}$ are formal real submanifolds through 0 in $\mathbb{C}^{N}$ and $\mathbb{C}^{N^{\prime}}$, respectively, then we say that the formal mapping $H$ maps $M$ into $M^{\prime}$, denoted $H:(M, 0) \rightarrow$ $\left(M^{\prime}, 0\right)$, if $\mathcal{I}\left(M^{\prime}\right) \subset \mathcal{H}_{*}(\mathcal{I}(M))$. (When $M$ and $M^{\prime}$ are real-analytic and $H$ is convergent, i.e. defines a holomorphic mapping near 0 , then $H(M) \subset M^{\prime}$ if and only if the formal mapping $H$ maps the formal submanifold $M$ into the formal submanifold $M^{\prime}$.) If $N=N^{\prime}$ and $H$ is invertible, then the ring homomorphism $\eta_{\mathcal{H}}: \mathbb{C}\left[\left[Z^{\prime}, \zeta^{\prime}\right]\right] \rightarrow \mathbb{C}[[Z, \zeta]]$, defined by (1.1.1), where $\left(Z^{\prime}, \zeta^{\prime}\right) \in \mathbb{C}^{N^{\prime}} \times \mathbb{C}^{N^{\prime}}$ and $(Z, \zeta) \in$ $\mathbb{C}^{N} \times \mathbb{C}^{N}$, is an isomorphism. If, in addition, $\operatorname{dim} M=\operatorname{dim} M^{\prime}$ and $H:(M, 0) \rightarrow$ $\left(M^{\prime}, 0\right)$, then $\mathcal{H}_{*}(\mathcal{I}(M))=\mathcal{I}\left(M^{\prime}\right)$ and $\eta_{\mathcal{H}}$ is an isomorphism between $\mathcal{I}(M)$ and $\mathcal{I}\left(M^{\prime}\right)$. In this case, we say that $M$ and $M^{\prime}$ are formally equivalent and that $H$ is a formal equivalence between $M$ and $M^{\prime}$.

In this paper, we shall consider formal mappings which are more general than formal equivalences. For a formal generic submanifold $M$ of codimension $d$ through the origin in $\mathbb{C}^{N}$, we define the Segre variety ideal $I_{1}(M) \subset \mathbb{C}[[Z]]$ as the ideal generated by $\rho_{1}(Z, 0), \ldots, \rho_{d}(Z, 0)$, where the $\rho_{j}(Z, \zeta)$ are generators of the ideal $\mathcal{I}(M) 11$ The ideal $I_{1}(M)$ is a manifold ideal which only depends on the ideal $\mathcal{I}(M)$ and not on the choice of generators $\rho_{j}(Z, \zeta)$. The reader can easily check that if $H:\left(\mathbb{C}^{N}, 0\right) \rightarrow\left(\mathbb{C}^{N^{\prime}}, 0\right)$ is a formal mapping which maps $M$ into another formal generic submanifold $M^{\prime}$ through the origin in $\mathbb{C}^{N^{\prime}}$, then in the notation introduced above, the homomorphism $\eta_{H}$ maps $I_{1}\left(M^{\prime}\right)$ into $I_{1}(M)$. Hence, $\eta_{H}$ induces a homomorphism

$$
\Phi_{H}: \mathbb{C}\left[\left[Z^{\prime}\right]\right] / I_{1}\left(M^{\prime}\right) \rightarrow \mathbb{C}[[Z]] / I_{1}(M) .
$$

The homomorphism $\Phi_{H}$ is called the Segre homomorphism of $H$. When $H$ is a formal equivalence, this homomorphism is an isomorphism. We shall consider in this paper formal mappings whose Segre homomorphisms are injective. In particular, all formal finite mappings satisfy this condition (see Lemma 6.1).

\footnotetext{
${ }^{1}$ In the convergent case, the variety defined by $I_{1}(M)$ in $\mathbb{C}^{N}$ is the so-called Segre variety of $M$ at 0 .
} 
A formal vector field in $\mathbb{C}^{N} \times \mathbb{C}^{N}$ of the form

$$
X=\sum_{j=1}^{N}\left(a_{j}(Z, \zeta) \frac{\partial}{\partial Z_{j}}+b_{j}(Z, \zeta) \frac{\partial}{\partial \zeta_{j}}\right)
$$

is said to be of type $(0,1)$ if $a_{j} \sim 0, j=1, \ldots, N$, and similarly of type $(1,0)$ if the $b_{j} \sim 0$. Let $M$ be a formal generic real submanifold in $\mathbb{C}^{N}$ of codimension $d$. We let $\mathcal{D}_{M}^{0,1}$ denote the $\mathbb{C}[[Z, \zeta]]$-module generated by all formal $(0,1)$ vector fields tangent to $M$. A formal vector field $L \in \mathcal{D}_{M}^{0,1}$ is usually called a $C R$ vector field tangent to $M$. We define $\mathcal{D}_{M}^{1,0}$ in a similar fashion. We define the $\mathbb{C}[[Z, \zeta]]$-module $\mathcal{D}_{M}$ by $\mathcal{D}_{M}=\mathcal{D}_{M}^{1,0} \oplus \mathcal{D}_{M}^{0,1}$, and define $\mathfrak{g}_{M}$ to be the Lie algebra generated by $\mathcal{D}_{M}$. We shall say that a collection $L_{1}, \ldots, L_{n} \in \mathcal{D}_{M}^{0,1}$, with $n=N-d$, is a basis for the $C R$ vector fields on $M$ if $\pi\left(L_{1}\right), \ldots, \pi\left(L_{n}\right)$ form a basis for the quotient module $\mathcal{D}_{M}^{0,1} / \mathcal{I}(M) \mathcal{D}_{M}^{0,1}$, where $\pi$ is the canonical projection $\pi: \mathcal{D}_{M}^{0,1} \rightarrow \mathcal{D}_{M}^{0,1} / \mathcal{I}(M) \mathcal{D}_{M}^{0,1}$. Loosely speaking, $L_{1}, \ldots, L_{n}$ form a basis for the CR vector fields on $M$ if they form a basis for $\mathcal{D}_{M}^{0,1}$ modulo those formal vector fields whose coefficients belong to $\mathcal{I}(M)$. (One can always find a basis for the CR vector fields on $M$; see $\S 1.2 .4$ below.)

Let $T_{0}^{\prime} \mathbb{C}^{2 N}$ denote the holomorphic tangent space of $\mathbb{C}^{2 N}$ at 0 , i.e. the space of all tangent vectors of the form

$$
\sum_{j=1}^{N}\left(\left.a_{j} \frac{\partial}{\partial Z_{j}}\right|_{0}+\left.b_{j} \frac{\partial}{\partial \zeta_{j}}\right|_{0}\right),
$$

with $a_{j}, b_{j} \in \mathbb{C}$. We denote by $\mathcal{D}_{M}(0)$ and $\mathfrak{g}_{M}(0)$ the subspaces of $T_{0}^{\prime} \mathbb{C}^{2 N}$ obtained by evaluating at 0 the coefficients of the formal vector fields in $\mathcal{D}_{M}$ and $\mathfrak{g}_{M}$ respectively. We say that $M$ is of finite type at 0 if $\operatorname{dim}_{\mathbb{C}} \mathfrak{g}_{M}(0)=\operatorname{dim} M=2 N-d$. (Note that the vector space $\mathcal{D}_{M}(0)$ has dimension $2 N-2 d$; this follows easily from the fact that $M$ is generic and of codimension $d$.)

Another notion which will be used in this paper is that of essential finiteness at 0 for a formal generic submanifold $M$ of codimension $d$ in $\mathbb{C}^{N}$. Let $L_{1}, \ldots, L_{n}$ be a basis for the $\mathrm{CR}$ vector fields on $M$ as described above. We write

$$
L_{j}=\sum_{k=1}^{N} a_{j k}(Z, \zeta) \frac{\partial}{\partial \zeta_{k}}
$$

where $a_{j k} \in \mathbb{C}[[Z, \zeta]]$, and set

$$
X_{j}:=\sum_{k=1}^{N} a_{j k}(0, \zeta) \frac{\partial}{\partial \zeta_{k}},
$$

for $j=1, \ldots, n$. Given generators $\rho_{1}(Z, \zeta), \ldots, \rho_{d}(Z, \zeta)$ for $\mathcal{I}(M)$, we define, for $l=1, \ldots, d$ and $\alpha \in \mathbb{Z}_{+}^{n}$,

$$
c_{l \alpha}(Z):=\left.X^{\alpha} \rho_{l}(Z, \zeta)\right|_{\zeta=0},
$$

with $X^{\alpha}:=X_{1}^{\alpha_{1}} \cdots X_{n}^{\alpha_{n}}$. We say that $M$ is essentially finite at 0 if the ideal generated by all the $c_{l \alpha}(Z)$ has finite codimension in $\mathbb{C}[[Z]]$. The reader can check that this definition depends only on the ideal $\mathcal{I}(M)$ and not on the choice of generators or basis of CR vector fields. (See [BER4, Chapter XI] for a similar definition for a smooth generic submanifold.) For a real-analytic generic submanifold, the notion of essential finiteness given above coincides with the standard one (see e.g. [BER4. 
Chapter XI]), since an ideal $\mathcal{J}$ in $\mathbb{C}\{Z\}$ is of finite codimension if and only if the ideal generated by $\mathcal{J}$ in $\mathbb{C}[[Z]]$ is of finite codimension (see e.g. BER4, Corollary 5.1.26]). Also, if $M$ is real-analytic and does not contain a nontrivial holomorphic subvariety through 0 , then $M$ is essentially finite at 0 (see [BER4, Chapter XI.4]). In fact, the analogous statement in the formal setting is also true. The proof of this relies on Lemma 3.32 below, and will be given in $\S 6$ below.

$\S 1.2$. Normal coordinates and the Segre mappings. We keep the notation introduced in the previous section; in particular, $M$ is a formal generic submanifold of codimension $d$ with real manifold ideal $\mathcal{I}=\mathcal{I}(M)$. There is a formal change of coordinates in $\mathbb{C}^{N}, Z=Z(z, w) \in \mathbb{C}[[z, w]]^{N}$, with $z=\left(z_{1}, \ldots, z_{n}\right)$, $w=\left(w_{1}, \ldots, w_{d}\right)$, and $N=n+d$, such that if we make the corresponding change of coordinates $\zeta=\bar{Z}(\chi, \tau)$, with $\chi=\left(\chi_{1}, \ldots, \chi_{n}\right), \tau=\left(\tau_{1}, \ldots \tau_{d}\right)$, then the ideal $\mathcal{I}$ of $M$, after making the above change of coordinates, is generated by

$$
w_{j}-Q_{j}(z, \chi, \tau), \quad j=1, \ldots, d,
$$

where the $Q_{j}(z, \chi, \tau) \in \mathbb{C}[[z, \chi, \tau]]$ satisfy

$$
Q_{j}(0, \chi, \tau) \sim Q_{j}(z, 0, \tau) \sim \tau_{j} .
$$

(See [BER4, Chapter IV].) Such coordinates will be called normal coordinates. It follows from the reality of $\mathcal{I}$ that $\mathcal{I}$ is also generated by

$$
\tau_{j}-\bar{Q}_{j}(\chi, z, w), \quad j=1, \ldots, d .
$$

In such coordinates, we can take

$$
L_{j}=\frac{\partial}{\partial \chi_{j}}+\sum_{l=1}^{d} \bar{Q}_{l, \chi_{j}}(\chi, z, w) \frac{\partial}{\partial \tau_{l}}, \quad j=1, \ldots, n,
$$

as a basis of the $\mathrm{CR}$ vector fields on $M$. The reader can easily check that if we write

$$
\bar{Q}_{j}(\chi, z, w)=\sum_{\alpha \in \mathbb{Z}_{+}^{n}} \bar{q}_{j \alpha}(z, w) \chi^{\alpha},
$$

then $M$ is essentially finite at 0 if and only if the ideal generated by $\left\{\bar{q}_{j \alpha}(z, 0)\right\}$, $j=1, \ldots, d, \alpha \in \mathbb{Z}_{+}^{n}$, has finite codimension in $\mathbb{C}[[z]]$.

We shall introduce some more notation. Let $M$ and $M^{\prime}$ be formal generic submanifolds through the origin in $\mathbb{C}^{N}$ and $\mathbb{C}^{N^{\prime}}$ of codimension $d$ and $d^{\prime}$, respectively. We let $Z=(z, w), \zeta=(\chi, \tau)$, with $z=\left(z_{1}, \ldots, z_{n}\right), w=\left(w_{1}, \ldots, w_{d}\right)$, $\chi=\left(\chi_{1}, \ldots, \chi_{n}\right)$ and $\tau=\left(\tau_{1}, \ldots, \tau_{d}\right)$, be formal normal coordinates for $M$, and similarly $Z^{\prime}=\left(z^{\prime}, w^{\prime}\right), \zeta^{\prime}=\left(\chi^{\prime}, \tau^{\prime}\right)$ be formal normal coordinates for $M^{\prime}$. Thus, the ideal $\mathcal{I}(M)$ is generated by (1.2.1), and similarly for $\mathcal{I}\left(M^{\prime}\right)$ with $Q$ replaced by $Q^{\prime}$. If $Z^{\prime}=H(Z)$ is a formal holomorphic mapping $\left(\mathbb{C}^{N}, 0\right) \rightarrow\left(\mathbb{C}^{N^{\prime}}, 0\right)$, sending $M$ into $M^{\prime}$, then we write $H=(F, G)$, with $F=\left(F_{1}, \ldots, F_{n^{\prime}}\right)$ and $G=\left(G_{1}, \ldots, G_{d^{\prime}}\right)$. The Segre homomorphism $\Phi_{H}: \mathbb{C}\left[\left[Z^{\prime}\right]\right] / I_{1}\left(M^{\prime}\right) \rightarrow \mathbb{C}\left[[Z] / I_{1}(M)\right.$ can be identified with the homomorphism

$$
\tilde{\Phi}: \mathbb{C}\left[\left[z^{\prime}\right]\right] \rightarrow \mathbb{C}[[z]],
$$

where $\tilde{\Phi}(h)(z)=h(F(z, 0))$ for $h \in \mathbb{C}\left[\left[z^{\prime}\right]\right]$. Thus, injectivity of the Segre homomorphism $\Phi_{H}$ is equivalent to the statement that there is no formal nontrivial power series $h \in \mathbb{C}\left[\left[z^{\prime}\right]\right]$ such that $h(F(z, 0)) \sim 0$. In particular, as mentioned in $\S 1.1$, if 
$H$ is a finite formal mapping (with $N=N^{\prime}$ and $d=d^{\prime}$ ), then $\Phi_{H}$ is injective (see Lemma 6.1).

We now introduce another important ingredient in this paper. Let $Q(z, \chi, \tau)$ be the $\mathbb{C}^{d}$-valued power series whose components are the $Q_{j}(z, \chi, \tau)$. Consider, for each integer $k \geq 1$, the formal mapping $v^{k}:\left(\mathbb{C}^{k n}, 0\right) \rightarrow\left(\mathbb{C}^{N}, 0\right)$ defined as follows. For $k=2 j$,

$$
\begin{array}{r}
v^{2 j}\left(z, \chi^{1}, \ldots, z^{j-1}, \chi^{j}\right):=\left(z, Q\left(z, \chi^{1}, \bar{Q}\left(\chi^{1}, z^{1}, Q\left(z^{1}, \chi^{2}, \ldots,\right.\right.\right.\right. \\
\left.\left.\left.\left.\bar{Q}\left(\chi^{j-1}, z^{j-1}, Q\left(z^{j-1}, \chi^{j}, 0\right)\right) \ldots\right)\right)\right)\right),
\end{array}
$$

and, for $k=2 j+1$,

$$
\begin{array}{r}
v^{2 j+1}\left(z, \chi^{1}, \ldots, z^{j-1}, \chi^{j}, z^{j}\right):=\left(z, Q\left(z, \chi^{1}, \bar{Q}\left(\chi^{1}, z^{1}, Q\left(z^{1}, \chi^{2}, \ldots,\right.\right.\right.\right. \\
\left.\left.\left.\left.Q\left(z^{j-1}, \chi^{j}, \bar{Q}\left(\chi^{j}, z^{j}, 0\right)\right) \ldots\right)\right)\right)\right),
\end{array}
$$

where $j=1,2, \ldots$ For $k=0,1$, we define

$$
v^{0}:=(0,0), \quad v^{1}(z):=(z, Q(z, 0,0))=(z, 0) .
$$

We shall refer to the mapping $v^{k}$ as the $k$ th Segre mapping of $M$.

Proposition 1.2.8. Let $f \in \mathcal{I}(M)$. Then, for any $k \geq 0$,

$$
f\left(v^{k+1}\left(z, \chi^{1}, z^{1}, \ldots\right), \bar{v}^{k}\left(\chi^{1}, z^{1}, \ldots\right)\right) \sim 0 .
$$

Proof. For simplicity, we only consider the case where $k=2 j$. It follows from (1.2.6) and (1.2.7) that

$$
v^{2 j+1}\left(z, \chi^{1}, \ldots, z^{j-1}, \chi^{j}, z^{j}\right) \sim\left(z, Q\left(z, \bar{v}^{2 j}\left(\chi^{1}, \ldots, z^{j-1}, \chi^{j}, z^{j}\right)\right)\right) .
$$

It suffices to show (1.2.9) for the generators of $\mathcal{I}(M)$ given by (1.2.1), for which (1.2.9) is an immediate consequence of (1.2.10).

In this paper, we shall not need the explicit form of the Segre mappings

$$
v^{k}:\left(\mathbb{C}^{k n}, 0\right) \rightarrow\left(\mathbb{C}^{N}, 0\right)
$$

given in (1.2.6) and (1.2.7), but merely the properties given by Proposition 1.2.8 and Theorem 1.2.11 below (and the fact that the $v^{k}$ are convergent when $M$ is real-analytic). It will be convenient to write $v^{k}(z, \xi), z \in \mathbb{C}^{n}$ and $\xi \in \mathbb{C}^{(k-1) n}$. Thus, for fixed $k$, we write $\xi=\left(\chi^{1}, z^{1}, \ldots\right) \in \mathbb{C}^{(k-1) n}$. The equation (1.2.9) can then be written as

$$
f\left(v^{k+1}(z, \xi), \bar{v}^{k}(\xi)\right) \sim 0,
$$

where, in $\left(1.2 .9^{\prime}\right)$, we have $\xi \in \mathbb{C}^{k n}$.

For a formal power series mapping $F:\left(\mathbb{C}^{\ell}, 0\right) \rightarrow\left(\mathbb{C}^{m}, 0\right)$ we denote by $\operatorname{rk}(F)$ the rank of the matrix $\left(\partial F_{j} / \partial x_{k}\right), j=1, \ldots, m, k=1, \ldots, \ell$, in $\mathbb{K}^{\ell}$; here, $\mathbb{K}$ denotes the quotient field of $\mathbb{C}[[x]]$. In particular, when $\ell=m$, then $\operatorname{rk}(F)=m$ if and only if the determinant of the matrix $\left(\partial F_{j} / \partial x_{k}\right)$, as a formal power series in $x$, is not identically zero. We shall need the following result. 
Theorem 1.2.11. Let $M$ be a formal generic submanifold of $\mathbb{C}^{N}$ through 0 of codimension $d$. Then, $M$ is of finite type at 0 if and only if there exists $k_{1} \leq d+1$ such that $\operatorname{rk}\left(v^{k}\right)=N$ for $k \geq k_{1}$. Moreover, if $M$ is real-analytic and of finite type at 0 , then there exists $\left(z_{0}, \xi_{0}\right) \in \mathbb{C}^{n} \times \mathbb{C}^{\left(2 k_{1}-1\right) n}$ arbitrarily close to the origin such that $v^{2 k_{1}}\left(z_{0}, \xi_{0}\right)=0$ and the rank of the Jacobian matrix $\partial v^{2 k_{1}} / \partial(z, \xi)$ at $\left(z_{0}, \xi_{0}\right)$ is $N$.

The proof of the first part of Theorem 1.2.11 can be found in [BER5] (see also BER1] and [BER4] for the case where $M$ is real-analytic). The proof of the last statement follows from [BER4, Proposition 10.6.19].

\section{§2. Statement of FURTher Results; examples}

We state now the main results of this paper from which the theorems in the introduction will be deduced. We begin with a result which will be shown to imply Theorem 3.

Theorem 2.1. Let $M$ and $M^{\prime}$ be real-analytic generic submanifolds through the origin in $\mathbb{C}^{N}$ and $\mathbb{C}^{N^{\prime}}$, respectively. Assume that $M$ is of finite type at 0 and that $M^{\prime}$ is essentially finite at 0 . If $H:\left(\mathbb{C}^{N}, 0\right) \rightarrow\left(\mathbb{C}^{N^{\prime}}, 0\right)$ is a formal holomorphic mapping sending $M$ into $M^{\prime}$ whose Segre homomorphism $\Phi_{H}$ is injective, then the formal series $H$ converges in a neighborhood of the origin.

We shall now give a result for hypersurfaces which in particular will imply Theorem 1.

Theorem 2.2. Let $M$ and $M^{\prime}$ be real-analytic hypersurfaces through the origin in $\mathbb{C}^{N}, N \geq 2$. Assume that $M$ is essentially finite at 0 and that $M^{\prime}$ does not contain any nontrivial holomorphic subvariety through 0 . Then any formal mapping $H:\left(\mathbb{C}^{N}, 0\right) \rightarrow\left(\mathbb{C}^{N}, 0\right)$ sending $M$ into $M^{\prime}$ is convergent.

Remark 2.3. The condition that $M^{\prime}$ above does not contain a nontrivial holomorphic subvariety is necessary for the conclusion of Theorem 2.2 to hold. Indeed, if $M^{\prime}$ is any real-analytic submanifold containing a nontrivial holomorphic subvariety through the origin in $\mathbb{C}^{N}$, then there exists a formal mapping $H:\left(\mathbb{C}^{N}, 0\right) \rightarrow\left(\mathbb{C}^{N}, 0\right)$ sending all of $\mathbb{C}^{N}$ into $M^{\prime}$ (even into the holomorphic subvariety contained in $M^{\prime}$ ) which does not converge. To see this, let $s \mapsto A(s)$ be a nontrivial holomorphic mapping from a neighborhood of the origin in the complex plane into the holomorphic subvariety contained in $M^{\prime}$ with $A(0)=0$. The reader can easily verify that there exists $f \in \mathbb{C}[[s]]$ with $f(0)=0$ such that at least one component of $A \circ f$ is not convergent. We may then take $H(Z)=A\left(f\left(Z_{1}\right)\right)$.

As a byproduct of the proof of Theorem 2.2, we obtain the following reflection principle for CR mappings between real-analytic hypersurfaces.

Theorem 2.4. Let $M$ and $M^{\prime}$ be real-analytic hypersurfaces through the origin in $\mathbb{C}^{N}, N \geq 2$. Assume that $M$ is essentially finite at 0 and $M^{\prime}$ does not contain any nontrivial holomorphic subvariety through 0 . Then every smooth CR mapping $h:(M, 0) \rightarrow\left(M^{\prime}, 0\right)$ which does not vanish to infinite order at 0 necessarily extends as a holomorphic mapping $\left(\mathbb{C}^{N}, 0\right) \rightarrow\left(\mathbb{C}^{N}, 0\right)$ sending $M$ into $M^{\prime}$.

In the case $N=2$, this result follows from the more general theorem of Huang $[\mathrm{Hu}]$ in which the only condition assumed on the CR mapping is continuity. However, for $N \geq 3$, Theorem 2.4 is new, since previous results in this context assumed 
more stringent conditions on the $\mathrm{CR}$ mapping. The reflection principle for realanalytic submanifolds in complex space has a long history; the reader is referred to the notes in [BER4, Chapter IX] for further information.

We return to the case of higher codimensional generic submanifolds. Theorem 5 will be a consequence of the following result on finite determination, or more precisely its corollary given below.

Theorem 2.5. Let $M$ and $M^{\prime}$ be formal generic submanifolds through the origin in $\mathbb{C}^{N}$ and $\mathbb{C}^{N^{\prime}}$, respectively. Assume that $M$ is of finite type at 0 , that $M^{\prime}$ is essentially finite at 0 , and that there exists a formal holomorphic mapping $H:\left(\mathbb{C}^{N}, 0\right) \rightarrow$ $\left(\mathbb{C}^{N^{\prime}}, 0\right)$ sending $M$ into $M^{\prime}$ whose Segre homomorphism $\Phi_{H}$ is injective. Then there exists an integer $K$ with the following property. If $H^{\prime}:\left(\mathbb{C}^{N}, 0\right) \rightarrow\left(\mathbb{C}^{N^{\prime}}, 0\right)$ is a formal holomorphic mapping sending $M$ into $M^{\prime}$ and if

$$
\frac{\partial^{|\alpha|} H^{\prime}}{\partial Z^{\alpha}}(0)=\frac{\partial^{|\alpha|} H}{\partial Z^{\alpha}}(0), \quad \forall|\alpha| \leq K,
$$

then $H \sim H^{\prime}$.

Corollary 2.7. Let $M$ be a formal generic submanifold through the origin in $\mathbb{C}^{N}$. Assume that $M$ is of finite type and essentially finite at 0 . Then there exists an integer $K$ with the following property. If $M^{\prime}$ is a formal generic submanifold through the origin in $\mathbb{C}^{N}$ of the same dimension as $M$, and if $H^{1}, H^{2}:\left(\mathbb{C}^{N}, 0\right) \rightarrow\left(\mathbb{C}^{N}, 0\right)$ are formal invertible mappings sending $M$ into $M^{\prime}$ which satisfy

$$
\frac{\partial^{|\alpha|} H^{1}}{\partial Z^{\alpha}}(0)=\frac{\partial^{|\alpha|} H^{2}}{\partial Z^{\alpha}}(0), \quad \forall|\alpha| \leq K,
$$

then $H^{1} \sim H^{2}$.

Proof of Corollary 2.7. We claim that it suffices to take $K$ to be the integer given by Theorem 2.5 with $M^{\prime}=M$ and $H(Z):=Z$. To see this, let $M^{\prime}, H^{1}, H^{2}$ be as in Corollary 2.7 and observe that if $(2.8)$ holds, then $\partial^{\alpha}\left(\left(H^{1}\right)^{-1} \circ H^{1}\right)(0)=$ $\partial^{\alpha}\left(\left(H^{1}\right)^{-1} \circ H^{2}\right)(0)$, for all $|\alpha| \leq K$. By Theorem 2.5 and the choice of $K$, we deduce that $\left(\left(H^{1}\right)^{-1} \circ H^{2}\right)(Z) \sim Z$ and, hence, the conclusion of Corollary 2.7.

We conclude this section by giving three simple examples motivating each of the three conditions in Theorem 2.1.

Example 2.9. Let $M=M^{\prime} \subset \mathbb{C}^{3}$ be the generic submanifold of codimension two defined by

$$
\operatorname{Im} w_{1}=|z|^{2}, \quad \operatorname{Im} w_{2}=0 .
$$

Observe that $M$ is essentially finite but not of finite type at 0 . Let $f \in \mathbb{R}\left[\left[w_{2}\right]\right]$ be nonconvergent with $f(0)=f^{\prime}(0)=0$. Then, the formal mapping $H:\left(\mathbb{C}^{3}, 0\right) \rightarrow$ $\left(\mathbb{C}^{3}, 0\right)$ defined by

$$
H\left(z, w_{1}, w_{2}\right):=\left(z, w_{1}, w_{2}+f\left(w_{2}\right)\right)
$$

is invertible, sends $M$ into $M$, but does not converge.

Example 2.12. Let $M=M^{\prime} \subset \mathbb{C}^{4}$ be the generic submanifold of codimension two defined by

$$
\operatorname{Im} w_{1}=\left|z_{1} z_{2}\right|^{2}, \quad \operatorname{Im} w_{2}=\left|z_{1} z_{2}\right|^{4} .
$$


In this case, $M$ is of finite type but not essentially finite at 0 . Let $f \in \mathbb{C}\left[\left[z_{1}\right]\right]$ be nonconvergent with $f(0)=0$. Then, the formal mapping $H:\left(\mathbb{C}^{4}, 0\right) \rightarrow\left(\mathbb{C}^{4}, 0\right)$ defined by

$$
H\left(z_{1}, z_{2}, w_{1}, w_{2}\right):=\left(z_{1} e^{f\left(z_{1}\right)}, z_{2} e^{-f\left(z_{1}\right)}, w_{1}, w_{2}\right)
$$

is invertible, sends $M$ into $M$, but does not converge.

Example 2.15. Let $M=M^{\prime} \subset \mathbb{C}^{4}$ be the generic submanifold of codimension two defined by

$$
\operatorname{Im} w_{1}=\left|z_{1}\right|^{2}-\left|z_{2}\right|^{2}, \quad \operatorname{Im} w_{2}=\left|z_{1}\right|^{4}-\left|z_{2}\right|^{4} .
$$

This time, $M$ is essentially finite and of finite type at 0 . Let $f \in \mathbb{C}\left[\left[z_{1}\right]\right]$ be nonconvergent with $f(0)=0$. Then, the formal mapping $H:\left(\mathbb{C}^{4}, 0\right) \rightarrow\left(\mathbb{C}^{4}, 0\right)$ defined by

$$
H\left(z_{1}, z_{2}, w_{1}, w_{2}\right):=\left(f\left(z_{1}\right), f\left(z_{1}\right), 0,0\right)
$$

sends $M$ into $M$, but does not converge. Observe that the Segre homomorphism $\Phi_{H}$ is not injective.

\section{§3. A REFLECTION IDENTITY}

The following reflection identity will play an important role in the proofs of Theorems 2.1 and 2.5.

Theorem 3.1. Let $M$ and $M^{\prime}$ be formal generic submanifolds through the origin in $\mathbb{C}^{N}$ and $\mathbb{C}^{N^{\prime}}$, respectively. Assume that $M^{\prime}$ is essentially finite at 0 . Let $H$ : $\left(\mathbb{C}^{N}, 0\right) \rightarrow\left(\mathbb{C}^{N^{\prime}}, 0\right)$ be a formal holomorphic mapping sending $M$ into $M^{\prime}$ whose Segre homomorphism $\Phi_{H}$ is injective. Then there exist a positive integer $r$, positive integers $N_{j}, j=1, \ldots, N^{\prime}$, and monic polynomials in $X$ of the form

$$
P_{j}\left(X, Z, \zeta,\left(a_{\gamma}\right)_{|\gamma| \leq r}\right):=X^{N_{j}}+\sum_{k=0}^{N_{j}-1} c^{j k}\left(Z, \zeta,\left(a_{\gamma}\right)_{|\gamma| \leq r}\right) X^{k}
$$

for $j=1, \ldots, N^{\prime}$, such that

$$
P_{j}\left(H_{j}(Z), Z, \zeta,\left(\partial^{\gamma} \bar{H}(\zeta)-\partial^{\gamma} \bar{H}(0)\right)_{|\gamma| \leq r}\right) \in \mathcal{I}(M) .
$$

Here, $\gamma \in \mathbb{Z}_{+}^{N}, a_{\gamma}=\left(a_{\gamma m}\right)_{1 \leq m \leq N^{\prime}}$, and the $c^{j k}$ are formal power series whose coefficients depend only on $M, M^{\prime}$ and on the values $\partial^{\beta} H(0)$ for $|\beta| \leq r$. In addition, if $H^{\prime}$ is another formal mapping $\left(\mathbb{C}^{N}, 0\right) \rightarrow\left(\mathbb{C}^{N^{\prime}}, 0\right)$ sending $M$ into $M^{\prime}$ and if $\partial^{\beta} H^{\prime}(0)=\partial^{\beta} H(0)$, for $|\beta| \leq r$, then (3.3) holds with $H$ replaced by $H^{\prime}$.

Moreover, if $M$ and $M^{\prime}$ are real-analytic, then each of the formal power series in (3.2) defines a holomorphic function in a neighborhood of the origin.

In order to prove Theorem 3.1, we let $Z=(z, w), \zeta=(\chi, \tau)$, with $z=$ $\left(z_{1}, \ldots, z_{n}\right), w=\left(w_{1}, \ldots, w_{d}\right), \chi=\left(\chi_{1}, \ldots, \chi_{n}\right)$ and $\tau=\left(\tau_{1}, \ldots, \tau_{d}\right)$, be formal normal coordinates for $M$, and similarly $Z^{\prime}=\left(z^{\prime}, w^{\prime}\right), \zeta^{\prime}=\left(\chi^{\prime}, \tau^{\prime}\right)$ be formal normal coordinates for $M^{\prime}$, as in $\S 1.2$. We shall first prove Theorem 3.1 in these coordinates. We let $L_{1}, \ldots, L_{n}$ be the basis for the formal $\mathrm{CR}$ vector fields on $M$ given by (1.2.4). Let $H=(F, G):\left(\mathbb{C}^{n} \times \mathbb{C}^{d}, 0\right) \rightarrow\left(\mathbb{C}^{n^{\prime}} \times \mathbb{C}^{d^{\prime}}, 0\right)$ be a formal holomorphic mapping sending $M$ into $M^{\prime}$. The following proposition gives the desired polynomial identities for the components of $F$. 
Proposition 3.4. Let $M, M^{\prime}$, and $H$ be as above. Assume that $M^{\prime}$ is essentially finite at 0 , and that the Segre homomorphism $\Phi_{H}$ is injective. Then there exist a positive integer $r$, positive integers $N_{j}, j=1, \ldots, n^{\prime}$, and monic polynomials

$$
P_{j}\left(X,\left(a_{\gamma}\right)_{|\gamma| \leq r},\left(b_{\gamma}\right)_{|\gamma| \leq r}\right):=X^{N_{j}}+\sum_{k=0}^{N_{j}-1} c^{j k}\left(\left(a_{\gamma}\right)_{|\gamma| \leq r},\left(b_{\gamma}\right)_{|\gamma| \leq r}\right) X^{k},
$$

for $j=1, \ldots, n^{\prime}$, such that

$$
P_{j}\left(F_{j}(z, w),\left(L^{\gamma} \bar{F}(\chi, \tau)-L^{\gamma} \bar{F}(0)\right)_{|\gamma| \leq r},\left(L^{\gamma} \bar{G}(\chi, \tau)\right)_{|\gamma| \leq r}\right) \in \mathcal{I}(M) .
$$

Here, $\gamma \in \mathbb{Z}_{+}^{n}, a_{\gamma}=\left(a_{\gamma m}\right)_{1 \leq m \leq n^{\prime}}, b_{\gamma}=\left(b_{\gamma l}\right)_{1 \leq l \leq d^{\prime}}$, and the $c^{j k}$ are formal power series whose coefficients depend only on $M^{\prime}$ and on the values $\left(L^{\beta} \bar{F}\right)(0)$ for $|\beta| \leq r$. In addition, if $H^{\prime}=\left(F^{\prime}, G^{\prime}\right)$ is another formal mapping $\left(\mathbb{C}^{N}, 0\right) \rightarrow\left(\mathbb{C}^{N^{\prime}}, 0\right)$ sending $M$ into $M^{\prime}$ and if $L^{\beta} \bar{F}^{\prime}(0)=L^{\beta} \bar{F}(0)$, for $|\beta| \leq r$, then (3.6) holds with $(F, G)$ replaced by $\left(F^{\prime}, G^{\prime}\right)$.

Moreover, if $M$ and $M^{\prime}$ are real-analytic, then each of the formal power series in (3.5) defines a holomorphic function in a neighborhood of the origin.

For the proof of Proposition 3.4, we shall need the following preliminary results.

Lemma 3.7. Let $p_{1}(X, Y, Z)$ and $p_{2}(X, Y, Z)$ be formal power series of the form

$$
\begin{aligned}
& p_{1}(X, Y, Z)=X^{N}+\sum_{j=0}^{N-1} a_{j}(Y, Z) X^{j}, \\
& p_{2}(X, Y, Z)=Y^{M}+K(X, Y, Z),
\end{aligned}
$$

where $X, Y \in \mathbb{C}, Z=\left(Z^{\prime}, Z^{\prime \prime}\right) \in \mathbb{C}^{k^{\prime}} \times \mathbb{C}^{k^{\prime \prime}}, a_{j}(0,0)=0$, and $K\left(X, Y, Z^{\prime}, 0\right) \sim 0$. Then the ideal $\mathcal{I}\left(p_{1}, p_{2}\right) \subset \mathbb{C}[[X, Y, Z]]$ contains a power series of the form

$$
r(Y, Z)=Y^{M N^{2}}+K^{\prime}(Y, Z),
$$

with $K^{\prime}\left(Y, Z^{\prime}, 0\right) \sim 0$.

Proof. For $\xi=\left(\xi_{1}, \ldots, \xi_{N}\right)$, we denote by $\sigma_{0}(\xi), \ldots, \sigma_{N-1}(\xi)$ the usual elementary symmetric polynomials of $\xi$ defined by

$$
\begin{array}{r}
\sigma_{N-1}(\xi)=-\sum_{j=1}^{N} \xi_{j}, \quad \sigma_{N-2}(\xi)=\sum_{1 \leq j<k \leq N} \xi_{j} \xi_{k}, \quad \ldots, \\
\sigma_{0}(\xi)=(-1)^{N} \prod_{j=1}^{N} \xi_{j} .
\end{array}
$$

Consider the ring

$$
\mathcal{R}=\mathbb{C}[[X, Y, Z, \xi]] / \mathcal{J}
$$

where $\mathcal{J}$ denotes the ideal generated by $\sigma_{j}(\xi)-a_{j}(Y, Z), j=0, \ldots, N-1$, where the $a_{j}$ are the coefficients of $p_{1}$ in (3.8). We use the notation $[f] \in \mathcal{R}$ for the equivalence class of a power series $f \in \mathbb{C}[[X, Y, Z, \xi]]$, and we also consider $\mathbb{C}[[X, Y, Z]]$ as a subring of $\mathbb{C}[[X, Y, Z, \xi]]$. Observe that

$$
\left[p_{1}\right]=\left[\prod_{j=1}^{N}\left(X-\xi_{j}\right)\right] .
$$


Consider the power series

$$
\hat{q}(Y, Z, \xi)=\prod_{j=1}^{N} p_{2}\left(\xi_{j}, Y, Z\right) .
$$

Since $\hat{q}$ is symmetric in the $\xi$, it follows from Newton's theorem for symmetric functions (see e.g. $[\mathrm{VW}])$ that there exists a power series $q\left(Y, Z, \sigma_{0}, \ldots, \sigma_{N-1}\right)$ such that

$$
\hat{q}(Y, Z, \xi)=q\left(Y, Z, \sigma_{0}(\xi), \ldots, \sigma_{N-1}(\xi)\right) .
$$

By the definition of the ideal $\mathcal{J}$, we have

$$
[\hat{q}(Y, Z, \xi)]=\left[q\left(Y, Z, a_{0}(Y, Z), \ldots, a_{N-1}(Y, Z)\right)\right] .
$$

We claim that $\left[q\left(Y, Z, a_{0}(Y, Z), \ldots, a_{N-1}(Y, Z)\right)^{N}\right]$ is in the ideal generated by $\left[p_{1}\right]$ and $\left[p_{2}\right]$ in $\mathcal{R}$. This is straightforward to check in view of (3.13-(3.16) since $\hat{q}^{N}$ is in the ideal generated by $p_{2}$ and $\prod_{j=1}^{N}\left(X-\xi_{j}\right)$ in $\mathbb{C}[[X, Y, Z, \xi]$. (The latter fact follows easily from the observation that, for any $j, \hat{q}$ is in the ideal generated by $p_{2}$ and $X-\xi_{j}$.)

It follows from the claim above that there are power series $c_{j}(X, Y, Z, \xi), j=1,2$, such that

$$
\begin{aligned}
q\left(Y, Z, a_{0}(Y, Z), \ldots, a_{N-1}(Y, Z)\right)^{N}-c_{1}(X, Y, Z, \xi) p_{1}(X, Y, Z) \\
-c_{2}(X, Y, Z, \xi) p_{2}(X, Y, Z) \in \mathcal{J} .
\end{aligned}
$$

We put $u(Y, Z):=q\left(Y, Z, a_{0}(Y, Z), \ldots, a_{N-1}(Y, Z)\right)$. It is easy to see that $u(Y, Z)$ is of the form $Y^{M N}+K^{\prime \prime}(Y, Z)$ with $K^{\prime \prime}\left(Y, Z^{\prime}, 0\right) \sim 0$. We shall complete the proof of Lemma 3.7 by showing that $r(Y, Z):=u(Y, Z)^{N}$ is in the ideal $\mathcal{I}\left(p_{1}, p_{2}\right)$. Since the generators of the ideal $\mathcal{J}$ are invariant under permutations of the components of $\xi$, we may assume without loss of generality that $c_{j}, j=1,2$, in (3.17) are symmetric in the $\xi$. Thus, again by Newton's theorem, there are power series $d_{j}(X, Y, Z, \sigma)$, $j=1,2, \sigma=\left(\sigma_{0}, \ldots, \sigma_{N-1}\right)$, such that

$$
\begin{aligned}
r(Y, Z)-d_{1}(X, Y, Z, & \left.\sigma_{0}(\xi), \ldots, \sigma_{N-1}(\xi)\right) p_{1}(X, Y, Z) \\
& -d_{2}\left(X, Y, Z, \sigma_{0}(\xi), \ldots, \sigma_{N-1}(\xi)\right) p_{2}(X, Y, Z) \in \mathcal{J}
\end{aligned}
$$

It follows that

$$
\begin{aligned}
r(Y, Z)-d_{1}\left(X, Y, Z, a_{0}(Y, Z), \ldots, a_{N-1}(Y, Z)\right) p_{1}(X, Y, Z) \\
-d_{2}\left(X, Y, Z, a_{0}(Y, Z), \ldots, a_{N-1}(Y, Z)\right) p_{2}(X, Y, Z) \in \mathcal{J} .
\end{aligned}
$$

Since the power series in (3.19) is independent of $\xi$, the desired fact that $r(Y, Z)$ is in $\mathcal{I}\left(p_{1}, p_{2}\right)$ will follow from the injectivity of the canonical homomorphism

$$
\mathbb{C}[[X, Y, Z]] \rightarrow \mathcal{R} .
$$

Thus, we must show that if $g(X, Y, Z) \in \mathcal{J}$, then $g \sim 0$. By expanding in $X$ and using the fact that the generators of $\mathcal{J}$ are independent of $X$, it suffices to prove that if

$$
f(Y, Z) \sim \sum_{j=0}^{N-1} e_{j}(Y, Z, \xi)\left(\sigma_{j}(\xi)-a_{j}(Y, Z)\right)
$$


for some power series $e_{j}$, then $f \sim 0$. By considering all possible formal curves $t \mapsto$ $(Y(t), Z(t))$ through the origin, we are reduced to proving the following statement: If

$$
h(t) \sim \sum_{j=0}^{N-1} \tilde{e}_{j}(t, \xi)\left(\sigma_{j}(\xi)-a_{j}(Y(t), Z(t))\right)
$$

for some power series $\tilde{e}_{j}$, then $h \sim 0$. By the formal Puiseux expansion (see e.g. BK] $)$, it follows that there exists an integer $J$ and formal series $f_{1}(w), \ldots, f_{N}(w)$ such that

$$
p_{1}\left(X, Y\left(w^{J}\right), Z\left(w^{J}\right)\right) \sim \prod_{j=1}^{N}\left(X-f_{j}(w)\right) .
$$

Making the substitutions $t=w^{J}$ and $\xi_{j}=f_{j}(w), j=1, \ldots, N$, in (3.21), we conclude that $h\left(w^{J}\right) \sim 0$ and hence $h \sim 0$. This completes the proof of Lemma 3.7 .

We shall make use of Lemma 3.7 to prove the following proposition.

Proposition 3.23. Let $f(u, v)=\left(f_{1}(u, v), \ldots, f_{r}(u, v)\right)$, with $u \in \mathbb{C}^{p}$ and $v \in$ $\mathbb{C}^{q}$, be formal power series with vanishing constant terms. Assume that the ideal generated by $f_{j}(0, v), j=1, \ldots, r$, has finite codimension in $\mathbb{C}[[v]]$. Then, there exist power series $P_{j}(u, v), j=1, \ldots, q$, of the form

$$
P_{j}(u, v)=v_{j}^{N_{j}}+\sum_{k=0}^{N_{j}-1} b_{j k}(u) v_{j}^{k}
$$

where the $b_{j k}$ are power series in $u$ with vanishing constant terms, such that each $P_{j}(u, v)$ is in the ideal $\mathcal{I}(f(u, v)) \subset \mathbb{C}[[u, v]]$.

Proof. We first reduce the situation to a simpler one. By standard arguments (see e.g. [BER4, Proposition 5.1.5]), there are power series $a_{j k}(v)$ and an integer $N$ such that

$$
v_{j}^{N} \sim \sum_{k=1}^{r} a_{j k}(v) f_{k}(0, v), \quad j=1, \ldots, q .
$$

Define power series $g_{k l}(u, v)$ such that

$$
f_{k}(u, v) \sim f_{k}(0, v)+\sum_{l=1}^{p} u_{l} g_{k l}(u, v), \quad k=1, \ldots, r .
$$

It follows that

$$
v_{j}^{N}+K_{j}(u, v) \sim \sum_{k=1}^{r} a_{j k}(v) f_{k}(u, v)
$$

where

$$
K_{j}(u, v):=\sum_{k=1}^{r} \sum_{l=1}^{p} a_{j k}(v) u_{l} g_{k l}(u, v), \quad j=1, \ldots, q .
$$

Observe that $v_{j}^{N}+K_{j}(u, v)$ are in the ideal $\mathcal{I}(f(u, v))$ and that $K_{j}(0, v) \sim 0$. 

form

Hence to prove Proposition 3.23 we may assume that $q=r$ and the $f_{j}$ are of the

$$
f_{j}(u, v):=v_{j}^{N}+K_{j}(u, v), \quad j=1, \ldots, q,
$$

where $K_{j}(0, v) \sim 0$. We reason by induction on $q$. For $q=1$, the desired conclusion follows from the (formal) Weierstrass Preparation Theorem (see e.g. [ZS]) applied to the power series $f_{1}(u, v)$, where $v=v_{1}$. We shall now show how to reduce the case of $q$ to that of $q-1$.

We apply the Weierstrass Preparation Theorem to $f_{1}(u, v)$. Hence there exist power series $c_{l}\left(u, v_{2}, \ldots, v_{q}\right), l=0, \ldots, N-1$, satisfying $c_{l}\left(0, v_{2}, \ldots, v_{n}\right) \sim 0$ so that

$$
f_{1}(u, v) \sim U(u, v)\left(v_{1}^{N}+\sum_{l=0}^{N-1} c_{l}\left(u, v_{2}, \ldots, v_{q}\right) v_{1}^{l}\right),
$$

where $U(u, v)$ is a unit in $\mathbb{C}[[u, v]]$. We apply, for $j=2, \ldots, q$, Lemma 3.7 with $X=v_{1}, Y=v_{j}, Z^{\prime \prime}=u, Z^{\prime}$ being the components $v_{k}$ for $k \geq 2$ and $k \neq j$, $p_{1}(X, Y, Z)$ being $v_{1}^{N}+\sum_{l=0}^{N-1} c_{l}\left(u, v_{2}, \ldots, v_{q}\right) v_{1}^{l}$, and $p_{2}(X, Y, Z)$ being $f_{j}(u, v)$. We conclude that the ideal $\mathcal{I}(f(u, v))$ contains power series of the form

$$
f_{j}^{\prime}\left(u, v_{2}, \ldots, v_{q}\right):=v_{j}^{N^{3}}+K_{j}^{\prime}\left(u, v_{2}, \ldots, v_{q}\right), \quad j=2, \ldots, q,
$$

with $K_{j}^{\prime}\left(0, v_{2}, \ldots, v_{q}\right) \sim 0$. By the inductive hypothesis, we conclude that $\mathcal{I}(f(u, v))$ contains power series $P_{j}\left(u, v_{j}\right), j=2, \ldots, q$, of the form (3.24). We obtain $P_{1}\left(u, v_{1}\right)$ by repeated application of Lemma 3.7 in a similar fashion as above. This completes the proof of Proposition 3.23.

For the proof of Proposition 3.4, we also need the following result which can be viewed as a formal version of the Nullstellensatz.

Lemma 3.32. Given $K(x)=\left(K_{1}(x), \ldots, K_{r}(x)\right)$ with $K_{j}(x) \in \mathbb{C}[[x]], x \in \mathbb{C}^{n}$, the following are equivalent:

(i) $\operatorname{dim}_{\mathbb{C}} \mathbb{C}[[x]] / \mathcal{I}(K(x))=\infty$,

(ii) there exist $\mu(s)=\left(\mu_{1}(s), \ldots, \mu_{n}(s)\right)$, with $\mu_{1}, \ldots, \mu_{n} \in \mathbb{C}[[s]]$ and $s \in \mathbb{C}$, such that $\mu(0)=0, \mu(s) \not 0$, and

$$
K_{j}(\mu(s)) \sim 0, \quad \forall 1 \leq j \leq r .
$$

Remark. The authors are indebted to C. Huneke for the proof of the implication (i) $\Rightarrow$ (ii).

Proof. (i) $\Rightarrow$ (ii). Since the ideal $\mathcal{I}(K(x))$ has infinite (vector space) codimension (i.e. (i) in the lemma is satisfied), there is a prime ideal $\mathfrak{p}$ containing $\mathcal{I}(K(x))$ that also has infinite codimension (see e.g. [BR, Lemma 3.3]). Since $\mathfrak{m}$, the maximal ideal in $\mathbb{C}[[x]]$, is the only proper prime ideal of finite codimension, we have $\mathfrak{p} \varsubsetneqq \mathfrak{m}$. We take $\mathfrak{p}$ to be maximal with this property, i.e. $\mathcal{I}(K(x)) \subset \mathfrak{p} \varsubsetneqq \mathfrak{m}$ and there is no prime ideal $\mathfrak{q}$ (of infinite codimension) such that $\mathfrak{p} \varsubsetneqq \mathfrak{q} \varsubsetneqq \mathfrak{m}$. Then the Krull dimension of $\mathbb{C}[[x]] / \mathfrak{p}$ is precisely 1 ([ZS, p. 218]). The integral closure of $\mathbb{C}[[x]] / \mathfrak{p}$ in its field of fractions is then a local 1-dimensional integrally closed ring, i.e. a complete discrete valuation ring denoted by $R$ below, whose residue field, being finite over $\mathbb{C}$, is precisely $\mathbb{C}$. It then follows from the Cohen Structure Theorem (see e.g. [ZS, p. $307])$ that $R$ is isomorphic to $\mathbb{C}[s]]$, with $s \in \mathbb{C}$. Thus, we obtain a homomorphism $\tilde{\psi}: \mathbb{C}[[x]] / \mathcal{I}(K(x)) \rightarrow \mathbb{C}[[s]]$ by composing the homomorphism $\mathbb{C}[[x]] / \mathcal{I}(K(x)) \rightarrow$ 
$\mathbb{C}[[x] / / \mathfrak{p}$, induced by the inclusion $\mathcal{I}(K(x)) \hookrightarrow \mathfrak{p}$, with the inclusion $\mathbb{C}[[x] / / \mathfrak{p} \hookrightarrow R \cong$ $\mathbb{C}[[s]]$. Let $\psi: \mathbb{C}[[x]] \rightarrow \mathbb{C}[[s]]$ be the homomorphism obtained by composing the projection $\mathbb{C}[[x]] \rightarrow \mathbb{C}[[x]] / \mathcal{I}(K(x))$ with $\tilde{\psi}$. By construction, this homomorphism is not identically 0 and $\mathcal{I}(K(x)) \subset$ ker $\psi$. The desired formal power series map $\mu(s)$ is given by $\mu_{j}(s)=\psi\left(x_{j}\right)$, which are not all 0 since $\psi$ is not identically 0 . To verify (3.33) it suffices to note that, for any $h \in \mathbb{C}[[x]]$, we have $\psi(h)(s)=h(\mu(s))$. The identity (3.33) follows from the fact that $\mathcal{I}(K(x)) \subset \operatorname{ker} \psi$.

(ii) $\Rightarrow(\mathbf{i})$. Assume, in order to reach a contradiction, that $\mathcal{I}(K(x))$ has finite codimension. Then there are integers $N_{j}$, for $1 \leq j \leq n$, such that $x_{j}^{N_{j}}$ is in $\mathcal{I}(K(x))$, i.e. there are $A_{j}^{k} \in \mathbb{C}[[x]](1 \leq j \leq n$ and $1 \leq k \leq r)$ such that

$$
x_{j}^{N_{j}} \sim \sum_{k=1}^{r} A_{j}^{k}(x) K_{k}(x) .
$$

Substituting $x=\mu(s)$ into this we obtain, using (3.33),

$$
\mu_{j}(s)^{N_{j}} \sim \sum_{k=1}^{r} A_{j}^{k}(\mu(s)) K_{k}(\mu(s)) \sim 0,
$$

for each $1 \leq j \leq n$. This contradicts the fact that the $\mu_{j}(s)$ are not all 0 , proving the desired implication.

Proof of Proposition 3.4. The fact that $H$ sends $M$ into $M^{\prime}$ is equivalent to

$$
\bar{G}(\chi, \tau)-\bar{Q}^{\prime}(\bar{F}(\chi, \tau), F(z, w), G(z, w)) \in \mathcal{I}(M)^{d^{\prime}} .
$$

In what follows, we write $F=F(z, w), \bar{F}=\bar{F}(\chi, \tau)$ and similarly for $G$ and $\bar{G}$. We write

$$
\bar{G}-\bar{Q}^{\prime}(\bar{F}, F, G)=\bar{G}-\bar{Q}^{\prime}(\bar{F}, F, 0)-P(\bar{F}, F, G) G \in \mathcal{I}(M)^{d^{\prime}},
$$

where $P$ is a $d^{\prime} \times d^{\prime}$ matrix of formal power series in $2 n^{\prime}+d^{\prime}$ variables. We decompose

$$
\bar{Q}^{\prime}(\bar{F}, F, 0)=\sum_{\alpha} \bar{Q}_{\alpha}^{\prime}(F) \bar{F}^{\alpha} .
$$

We claim that the ideal $\mathcal{J} \subset \mathbb{C}[[\mu]]$ generated by $\sum_{\alpha} \bar{Q}_{\alpha}^{\prime}(\mu) L^{\beta} \bar{F}^{\alpha}(0), \beta \in \mathbb{Z}_{+}^{n}$, has finite codimension. To see this, first observe that

$$
L^{\beta} h(0):=\left.L^{\beta} h(\chi, \tau)\right|_{0}=\frac{\partial^{|\beta|} h}{\partial \chi^{\beta}}(0),
$$

for any power series $h \in \mathbb{C}[[\chi, \tau]]$. Now, pick an integer $r$ such that the ideal $\mathcal{J}$ is generated by the power series $\sum_{\alpha} \bar{Q}_{\alpha}^{\prime}(\mu) L^{\beta} \bar{F}^{\alpha}(0)$ for $|\beta| \leq r$. Recall that, in normal coordinates, injectivity of the Segre homomorphism $\Phi_{H}$ is equivalent to the fact that there are no nontrivial power series $h \in \mathbb{C}\left[\left[\chi^{\prime}\right]\right]$ such that $h(\bar{F}(\chi, 0)) \sim 0$. Hence, there are no nontrivial power series $g \in \mathbb{C}\left[\left[s, \chi^{\prime}\right]\right]$ such that $g(s, \bar{F}(\chi, 0)) \sim 0$. It follows that for any formal curve $s \mapsto \mu(s)$ with $\mu=\left(\mu_{1}, \ldots, \mu_{n}\right), \mu(0)=0$, and $\mu \nsim 0$, we have

$$
\sum_{\alpha} \bar{Q}_{\alpha}^{\prime}(\mu(s)) \bar{F}(\chi, 0)^{\alpha} \nsim 0
$$

because otherwise $\bar{Q}_{\alpha}^{\prime}(\mu(s)) \sim 0$ for all $\alpha \in \mathbb{Z}_{+}^{n^{\prime}}$ which would contradict the essential finiteness of $M^{\prime}$ by the implication (ii) $\Longrightarrow$ (i) in Lemma 3.32. (We have used here the characterization of essential finiteness in normal coordinates given in §1.2.) It 
follows by using (3.39) that, given a formal curve $\mu(s)$ as above, there exists $\beta \in \mathbb{Z}_{+}^{n}$ such that

$$
\sum_{\alpha} \bar{Q}_{\alpha}^{\prime}(\mu(s)) L^{\beta} \bar{F}^{\alpha}(0) \not 0
$$

By the choice of $r$ above, we may assume that $|\beta| \leq r$. The claim follows by applying the implication (i) $\Longrightarrow$ (ii) in Lemma 3.32 to the collection of power series $\sum_{\alpha} \bar{Q}_{\alpha}^{\prime}(\mu) L^{\beta} \bar{F}^{\alpha}(0),|\beta| \leq r$.

We now apply $L^{\beta},|\beta| \leq r$, to $(3.37)$ and substitute $G=Q^{\prime}(F, \bar{F}, \bar{G})$ to obtain

$$
\sum_{\alpha} \bar{Q}_{\alpha}^{\prime}(F) L^{\beta} \bar{F}^{\alpha}+\left.L^{\beta}(P(\bar{F}, F, G)) G\right|_{G=Q^{\prime}(F, \bar{F}, \bar{G})}-L^{\beta} \bar{G} \in \mathcal{I}(M)^{d^{\prime}} .
$$

Observe that $L^{\beta} \bar{G}(0)=0$, by normality of the coordinates, but $L^{\beta} \bar{F}(0)$ is in general not 0 . We can rewrite $(3.42)$ as

$$
\begin{aligned}
& \sum_{\alpha} \bar{Q}_{\alpha}^{\prime}(F)\left(L^{\beta} \bar{F}^{\alpha}-L^{\beta} \bar{F}(0)^{\alpha}\right)+\sum_{\alpha} \bar{Q}_{\alpha}^{\prime}(F) L^{\beta} \bar{F}(0)^{\alpha} \\
& \quad+S\left(F, \bar{F}, \bar{G},\left(L^{\gamma} \bar{F}-L^{\gamma} \bar{F}(0)\right)_{1 \leq|\gamma| \leq r}\right) Q^{\prime}(F, \bar{F}, \bar{G})-L^{\beta} \bar{G} \in \mathcal{I}(M)^{d^{\prime}},
\end{aligned}
$$

for some formal power series $S$ which depends on $M^{\prime}$ and the values $L^{\gamma} \bar{F}(0)$ for $|\gamma| \leq r$. (We have used here the fact that $L^{\beta}(P(\bar{F}, F, G))$ is polynomial in the variables $L^{\gamma} \bar{F}$ for $1 \leq|\gamma| \leq r$. $)$ Let $a_{\gamma}=\left(a_{\gamma p}\right)_{1 \leq p \leq n^{\prime}}=\left(L^{\gamma} \bar{F}_{p}-L^{\gamma} \bar{F}_{p}(0)\right)_{1 \leq p \leq n^{\prime}}$ and $b_{\gamma}=\left(b_{\gamma l}\right)_{1 \leq l \leq d^{\prime}}=\left(L^{\gamma} \bar{G}_{l}\right)_{1 \leq l \leq d^{\prime}}$, considered as independent variables, for $|\gamma| \leq r$. We write $a=\left(a_{\gamma p}\right)$ and $b=\left(b_{\gamma l}\right)$. The equations (3.43) can be written in the form

$$
R_{\beta j}\left(\left(L^{\gamma} \bar{F}_{p}-L^{\gamma} \bar{F}_{p}(0)\right),\left(L^{\gamma} \bar{G}_{l}\right), F\right) \in \mathcal{I}(M), \quad j=1, \ldots, d^{\prime},|\beta| \leq r,
$$

where the $R_{\beta j}$ are formal power series whose coefficients depend only on $M^{\prime}$ and the values $L^{\beta} \bar{F}(0)$ for $|\beta| \leq r$. Furthermore, using the normality of the coordinates, we observe that $R_{\beta}(0,0, F):=\left(R_{\beta j}(0,0, F)\right)_{1 \leq j \leq d^{\prime}} \sim \sum_{\alpha} \bar{Q}_{\alpha}^{\prime}(F) L^{\beta} \bar{F}(0)^{\alpha}$. Thus, the ideal generated by $R_{\beta j}(0,0, \mu), j=1, \ldots, \bar{d}^{\prime}$ and $|\beta| \leq r$, has finite codimension by the claim above. The first part of Proposition 3.4 then follows from Proposition 3.23 applied to the power series $R_{\beta j}(a, b, F)$ (where $(a, b, F)$ are considered as independent variables). Moreover, if $H^{\prime}=\left(F^{\prime}, G^{\prime}\right)$ is another formal mapping sending $M$ into $M^{\prime}$ and if $L^{\beta} \bar{F}^{\prime}(0)=L^{\beta} \bar{F}(0)$, for $|\beta| \leq r$, then the ideal generated by the power series $\sum_{\alpha} \bar{Q}_{\alpha}^{\prime}(\mu) L^{\beta} \bar{F}^{\prime}(0)$, for $|\beta| \leq r$, has finite codimension in $\mathbb{C}[[\mu]]$. The fact that $H^{\prime}$ satisfies the same polynomial identity as $H$ follows from the construction above.

To prove the last statement of Proposition 3.4, i.e. when $M$ and $M^{\prime}$ are assumed to be real-analytic, we observe that both Proposition 3.23 and Lemma 3.32 (which are used in the proof above) hold in the convergent setting. Indeed, for the proof of Proposition 3.23 in the convergent case the reader is referred to BER4, Theorem 5.3.9]. Lemma 3.32 in the convergent case is a special case of the Nullstellensatz for convergent power series (see e.g. Gu Theorem II.E.2]). The convergence of the power series in (3.5) now follows by an inspection of the proof above in the formal case. This completes the proof of Proposition 3.4.

Proof of Theorem 3.1. To complete the proof of Theorem 3.1, using Proposition 3.4 , we observe that

$$
G(z, w)-Q^{\prime}(F(z, w), \bar{F}(\chi, \tau,) \bar{G}(\chi, \tau)) \in \mathcal{I}(M)^{d^{\prime}} .
$$


We obtain polynomial identities for the components $G$, similar to those for $F$, by repeated applications of Lemma 3.7 as in the proof of Proposition 3.23. To obtain the identities in (3.3), it suffices to replace the vector fields $L_{1}, \ldots, L_{n}$ in the identities (3.6) for $F$, and the similar ones just obtained for $G$, by their expressions in the coordinates $(z, w, \chi, \tau)$. The fact that Theorem 3.1 holds in any set of coordinates follows from the result in normal coordinates by an application of Proposition 3.23. This completes the proof of Theorem 3.1.

\section{$\S 4$. Proof of Theorem 2.1}

For the proof of Theorem 2.1, we shall make use of Theorem 3.1 and the following consequence of the approximation theorem of Artin (see [A]).

Theorem 4.1. Let $h \in \mathbb{C}[[x]], x=\left(x_{1}, \ldots, x_{m}\right)$, and assume that $P(h(x), x) \sim 0$, where

$$
P(X, x)=\sum_{j=0}^{J} a_{j}(x) X^{j}, \quad a_{j} \in \mathbb{C}\{x\}, a_{J}(x) \not \equiv 0 .
$$

Then $h \in \mathbb{C}\{x\}$. Here, $\mathbb{C}\{x\}$ denotes the ring of convergent power series in $x$.

Proof of Theorem 4.1. By Artin's approximation theorem, for every integer $k$, there exists a convergent solution $X=\tilde{h}_{k}(x)$ of $P(X, x)=0$ such that the power series of $\tilde{h}_{k}(x)$ agrees with that of $h(x)$ up to order $k$. The conclusion in Theorem 4.1 is a consequence of Lemma 4.3 below.

Lemma 4.3. Let $P(X, x)$ be of the form

$$
P(X, x)=\sum_{j=0}^{J} a_{j}(x) X^{j}, \quad a_{j} \in \mathbb{C}[[x]], a_{J}(x) \not \equiv 0 .
$$

Then there exists a positive integer $m(P)$ such that if $h, h^{\prime} \in \mathbb{C}[[x]]$ satisfy

$$
\begin{aligned}
& P(h(x), x) \sim P\left(h^{\prime}(x), x\right) \sim 0, \\
& \partial^{\alpha} h(0)=\partial^{\alpha} h^{\prime}(0), \quad \forall|\alpha| \leq m(P),
\end{aligned}
$$

then $h(x) \sim h^{\prime}(x)$.

Proof. The polynomial in $X, P(X, x)$, has at most $J$ distinct formal power series roots. The desired number $m(P)$ is determined by these roots, as the reader can easily check.

Proof of Theorem 2.1. Consider the following vector fields tangent to $M$ near the origin:

$$
S_{j}=\frac{\partial}{\partial Z_{j}}-\rho_{Z_{j}}(Z, \zeta)\left(\rho_{\zeta^{\prime \prime}}(Z, \zeta)\right)^{-1} \frac{\partial}{\partial \zeta^{\prime \prime}}, \quad j=1, \ldots, N,
$$

where $\rho=\left(\rho_{1}, \ldots, \rho_{d}\right)$ is a defining $\mathbb{R}^{d}$-valued function for $M$ near 0 with $\rho_{\zeta^{\prime \prime}}=$ $\left(\partial \rho_{j} / \partial \zeta_{k}\right)_{1 \leq j, k \leq d}$ invertible at 0 (which we may assume without loss of generality); in (4.6), $\partial / \partial \zeta^{\prime \prime}=\left(\partial / \partial \zeta_{k}\right)_{1 \leq k \leq d}$ is considered as a $d \times 1$ matrix and $\rho_{Z_{j}}=$ $\left(\rho_{k, Z_{j}}\right)_{1 \leq k \leq d}$ as a $1 \times d$ matrix. Observe that $S^{\alpha} h=\partial^{|\alpha|} h / \partial Z^{\alpha}$ for any $h \in \mathbb{C}[[Z]]$.

We shall make use of the Segre mappings of $M$ introduced in $\S 1.2$. Recall the notation $v^{k+1}(z, \xi), z \in \mathbb{C}^{n}$ and $\xi \in \mathbb{C}^{(k-1) n}$, for the $(k+1)$ th Segre mapping of 
$M$ at 0 . (We use the notation $v^{0}$ for the constant mapping $v^{0}=0$.) Consider the following property, for $k \geq 0$ and $\alpha \in \mathbb{Z}_{+}^{N}$ :

$(*)_{k, \alpha}$

$$
\begin{aligned}
& \left(\partial^{\beta} H\right) \circ v^{l} \text { is convergent, } \quad \forall 0 \leq l \leq k-1, \forall \beta \in \mathbb{Z}_{+}^{N}, \\
& \left(\partial^{\beta} H\right) \circ v^{k} \text { is convergent, } \quad \forall \beta \leq \alpha,
\end{aligned}
$$

where $\beta<\alpha$ means that $\beta$ precedes $\alpha$ in the lexicographical ordering of $\mathbb{Z}_{+}^{N}$. We shall say that $(*)_{k}$ holds if $(*)_{k, \alpha}$ holds for all $\alpha \in \mathbb{Z}_{+}^{N}$. We shall prove $(*)_{k}$ for all $k$ by induction. Observe that $(*)_{0}$ holds, since $v^{0}$ is the constant mapping. We assume $(*)_{k}$, and wish to prove $(*)_{k+1}$. We first prove $(*)_{k+1,0}$. We shall make use of Theorem 3.1. We substitute $(Z, \zeta)=\left(v^{k+1}(z, \xi), \bar{v}^{k}(\xi)\right)$ in (3.3). By Proposition 1.2.8, we have for $j=1, \ldots, N^{\prime}$

$$
P_{j}\left(H_{j}\left(v^{k+1}(z, \xi)\right), v^{k+1}(z, \xi), \bar{v}^{k}(\xi),\left(\partial^{\gamma} \bar{H}\left(\bar{v}^{k}(\xi)\right)-\partial^{\gamma} \bar{H}(0)\right)_{|\gamma| \leq r}\right) \sim 0 .
$$

It follows from the inductive hypothesis $(*)_{k}$ that

$$
P_{j}\left(X, v^{k+1}(z, \xi), \bar{v}^{k}(\xi),\left(\partial^{\gamma} \bar{H}\left(\bar{v}^{k}(\xi)\right)-\partial^{\gamma} \bar{H}(0)\right)_{|\gamma| \leq r}\right) \in \mathbb{C}\{z, \xi\}[X] .
$$

Hence, by Theorem 4.1 and $(4.7)$, we conclude that $(*)_{k+1,0}$ holds. To complete the proof of $(*)_{k+1}$, we prove $(*)_{k+1, \alpha}$ for all $\alpha$ by induction on $\alpha$ (using the lexicographic ordering of $\mathbb{Z}_{+}^{N}$ ). For this we shall need the following result whose proof will be given later. Recall that if $\mathcal{J} \subset \mathbb{C}[[x]]$ is an ideal, then we say that a formal vector field $S$ is tangent to $\mathcal{J}$ if $S$ is a derivation of $\mathbb{C}[[x]]$ preserving $\mathcal{J}$.

Lemma 4.9. Let $\mathcal{J} \subset \mathbb{C}[[x]], x=\left(x_{1}, \ldots, x_{m}\right)$, be an ideal and $S_{1}, \ldots, S_{N}$ formal vector fields tangent to $\mathcal{J}$. Assume that $h \in \mathbb{C}[[x]]$, and that there exists a formal power series $P(X, x)$ of the form

$$
P(X, x)=\sum_{j=0}^{J} a_{j}(x) X^{j}, \quad a_{j} \in \mathbb{C}[[x]], a_{J}(x) \sim 1,
$$

with $P(h(x), x) \in \mathcal{J}$. Then, for any formal mapping $v:\left(\mathbb{C}^{r}, 0\right) \rightarrow\left(\mathbb{C}^{m}, 0\right)$ such that $f \circ v \sim 0$ for all $f \in \mathcal{J}$ and any $\alpha \in \mathbb{Z}_{+}^{N}$, there exists a formal power series $R_{\alpha}(X, x)$ of the form

$$
R_{\alpha}(X, x)=\sum_{j=0}^{J} b_{\alpha j}(x) X^{j}, \quad b_{\alpha j} \in \mathbb{C}[[x]],
$$

with $R_{\alpha}(X, v(y)) \nsim 0$ and

$$
R_{\alpha}\left(\left(S^{\alpha} h\right)(v(y)), v(y)\right) \sim 0
$$

Here, we have used the notation $S^{\alpha}=S_{1}^{\alpha_{1}} \cdots S_{N}^{\alpha_{N}}$. Furthermore, the coefficients $b_{\alpha j}$ are universal polynomials in $S^{\beta} a_{j},|\beta| \leq|\alpha| J$ and $j=0, \ldots, J-1$, and $S^{\gamma} h$, for $\gamma<\alpha$, where $\gamma<\alpha$ means that $\gamma$ precedes $\alpha$ in the lexicographic ordering of $\mathbb{Z}_{+}^{N}$.

We proceed with the proof of Theorem 2.1. Assume $(*)_{k+1, \alpha^{0}}$ for some $\alpha^{0} \in \mathbb{Z}_{+}^{N}$. Recall that $(*)_{k+1,0}$ has already been proved. We complete the induction by proving $(*)_{k+1, \alpha^{\prime}}$, where $\alpha^{\prime}$ is the multi-index immediately following $\alpha^{0}$ in the lexicographic ordering of $\mathbb{Z}_{+}^{N}$.

By applying Lemma 4.9 to the polynomials

$$
P_{j}\left(X, Z, \zeta,\left(\partial^{\gamma} \bar{H}(\zeta)-\partial^{\gamma} \bar{H}(0)\right)_{|\gamma| \leq r}\right), \quad j=1, \ldots, N^{\prime},
$$


with $\mathcal{J}=\mathcal{I}(M), S_{j}$ given by (4.6), $x=(Z, \zeta), h=H_{j}$, and $v=\left(v^{k+1}, \bar{v}^{k}\right)$, we obtain, for each $j=1, \ldots, N^{\prime}$ and every $\alpha \in \mathbb{Z}_{+}^{N}$, power series $R_{j \alpha}(X, Z, \zeta)$ in $\mathbb{C}[[Z, \zeta]][X]$ such that $R_{j \alpha}\left(X, v^{k+1}(z, \xi), \bar{v}^{k}(\xi)\right) \nsim 0$ and

$$
R_{j \alpha}\left(\partial^{\alpha} H_{j}\left(v^{k+1}(z, \xi)\right), v^{k+1}(z, \xi), \bar{v}^{k}(\xi)\right) \sim 0 .
$$

Moreover, the coefficients of $R_{j \alpha}$, as a polynomial in $X$, are polynomials in $\partial^{\beta} H_{j}(Z)$, for $\beta<\alpha$, and $S^{\gamma} c^{j l}\left(Z, \zeta,\left(\partial^{\delta} \bar{H}_{j}(\zeta)-\partial^{\delta} \bar{H}_{j}(0)\right)_{|\delta| \leq r}\right), \gamma \leq N_{j} \alpha$, where $c^{j l}$ are given by (3.2). Thus, by the inductive hypothesis $(*)_{k+1, \alpha^{0}}$, it follows that

$$
R_{j \alpha^{\prime}}\left(X, v^{k+1}(z, \xi), \bar{v}^{k}(\xi)\right) \in \mathbb{C}\{z, \xi\}[X] .
$$

The property $(*)_{k+1, \alpha^{\prime}}$ follows from Theorem 4.1. This completes the proof of property $(*)_{k}$ for all $k$.

We shall apply $(*)_{k, 0}$ for $k=2 k_{1}$, where $k_{1}$ is given by Theorem 1.2.11. By Theorem 1.2.11, there exists $\left(z^{0}, \xi^{0}\right) \in \mathbb{C}^{n} \times \mathbb{C}^{\left(2 k_{1}-1\right) n}$, arbitrarily close to $(0,0)$, such that the rank of $\partial v^{2 k_{1}} / \partial(z, \xi)$ at $\left(z^{0}, \xi^{0}\right)$ is $N$ and $v^{2 k_{1}}\left(z^{0}, \xi^{0}\right)=0$. Since $(z, \xi) \mapsto H\left(v^{2 k_{1}}(z, \xi)\right)$ is holomorphic in a neighborhood of $(0,0)$ by $(*)_{2 k_{1}}$, we may choose $\left(z^{0}, \xi^{0}\right)$ in that neighborhood. By applying the implicit function theorem, we may find a right inverse of $v^{2 k_{1}}, Z \mapsto \theta(Z)$ with $\theta(0)=\left(z^{0}, \xi^{0}\right)$. Hence, $H\left(v^{2 k_{1}}(\theta(Z))\right)$ is a convergent mapping in a neighborhood of 0 . This completes the proof of Theorem 2.1 modulo the proof of Lemma 4.9, since $v^{2 k_{1}}(\theta(Z)) \equiv Z$ near $0 \in \mathbb{C}^{N}$.

Proof of Lemma 4.9. Observe that (4.7) holds for $\alpha=0$ with $R_{0}(X, x)=P(X, x)$. We will need the following Leibnitz formula:

$$
S^{\gamma} P(h(x), x)=\sum_{k=0}^{J} \sum_{\mu+\nu^{1}+\cdots+\nu^{k}=\gamma} \frac{\gamma !}{\mu ! \nu^{1} ! \cdots \nu^{k !}}\left(S^{\mu} a_{k}\right)\left(S^{\nu^{1}} h\right) \cdots\left(S^{\nu^{k}} h\right) .
$$

The reader can check that (4.14) can be rewritten as follows. For any given $\alpha \leq \gamma$,

$$
\begin{aligned}
& S^{\gamma} P(h(x), x)= \\
& A(\gamma, \alpha, 0)+\sum_{j=1}^{J} \sum_{\substack{\nu^{1} \geq \alpha, \ldots, \nu^{j} \geq \alpha \\
\gamma-\nu^{1}-\cdots-\nu^{j} \in \mathbb{Z}_{+}^{N}}} A\left(\gamma, \alpha, j, \nu^{1}, \ldots, \nu^{j}\right)\left(S^{\nu^{1}} h\right) \cdots\left(S^{\nu^{j}} h\right),
\end{aligned}
$$

where

$$
\begin{aligned}
& A\left(\gamma, \alpha, j, \nu^{1}, \ldots, \nu^{j}\right)= \\
& \sum_{k=j}^{J}\left(\begin{array}{c}
k \\
j
\end{array}\right) \sum_{\substack{\mu, \nu^{j+1}, \ldots, \nu^{k} \\
\mu+\nu^{1}+\cdots+\nu^{k}=\gamma \\
\nu^{j+1}<\alpha, \ldots, \nu^{k}<\alpha}} \frac{\gamma !}{\mu ! \nu^{1} ! \cdots \nu^{k !}}\left(S^{\mu} a_{k}\right)\left(S^{\nu^{j+1}} h\right) \cdots\left(S^{\nu^{k}} h\right), \\
& A(\gamma, \alpha, 0)=\sum_{k=0}^{J} \sum_{\substack{\mu+\nu^{1}+\cdots+\nu^{k}=\gamma \\
\nu^{1}<\alpha, \ldots, \nu^{k}<\alpha}} \frac{\gamma !}{\mu ! \nu^{1} ! \cdots \nu^{k !}}\left(S^{\mu} a_{k}\right)\left(S^{\nu^{j+1}} h\right) \cdots\left(S^{\nu^{k}} h\right) .
\end{aligned}
$$


Observe that the first term in the sum in (4.16) (i.e. the term corresponding to $k=j)$ is

$$
\frac{\gamma !}{\left(\gamma-\nu^{1}-\cdots-\nu^{j}\right) ! \nu^{1} ! \cdots \nu^{j !}} S^{\gamma-\nu^{1}-\cdots-\nu^{j}} a_{j} .
$$

For $\gamma=\alpha$, we have, from (4.15),

$$
S^{\alpha} P(h(x), x)=A(\alpha, \alpha, 0)+A(\alpha, \alpha, 1, \alpha) S^{\alpha} h .
$$

If $A(\alpha, \alpha, 1, \alpha) \circ v \not \alpha 0$, then we take $R_{\alpha}(X, x)=A(\alpha, \alpha, 0)+A(\alpha, \alpha, 1, \alpha) X$. If not, then we denote by $\gamma^{0}$ the first multi-index $>\alpha$ for which $A\left(\gamma^{0}, \alpha, j, \nu^{1}, \ldots, \nu^{j}\right) \circ v \chi$ 0 for some $1 \leq j \leq J, \nu^{1} \geq \alpha, \ldots, \nu^{j} \geq \alpha$. Such a $\gamma^{0}$ exists (and is $\leq J \alpha$ ), since $A(J \alpha, \alpha, J, \alpha, \ldots, \alpha) \sim(J \alpha) ! /(\alpha !)^{J}$ as is easily verified. We claim that necessarily $A\left(\gamma^{0}, \alpha, j_{0}, \alpha, \ldots, \alpha\right) \circ v \not 0$ for some $1 \leq j_{0} \leq J$ and $A\left(\gamma^{0}, \alpha, j, \nu^{1}, \ldots, \nu^{j}\right) \circ v \sim 0$ for all $1 \leq j \leq J$ and $\nu^{1} \geq \alpha, \ldots, \nu^{j} \geq \alpha$ such that $\sum_{1 \leq l \leq j} \nu^{l}>j \alpha$. Indeed, the following identity is a consequence of (4.16):

$$
A\left(\gamma^{0}, \alpha, j, \nu^{1}, \ldots, \nu^{j}\right)=e_{\gamma^{0}, \alpha, j, \nu^{1}, \ldots, \nu^{j}} A\left(\gamma^{1}, \alpha, j, \alpha, \ldots, \alpha\right),
$$

where

$$
\gamma^{1}=\gamma^{0}+j \alpha-\sum_{l=1}^{j} \nu^{l}
$$

and $e_{\gamma^{0}, \alpha, j, \nu^{1}, \ldots, \nu^{j}}$ is a positive number depending only on $\gamma^{0}, \alpha, j, \nu^{1}, \ldots, \nu^{j}$. Observe that $\gamma^{1} \geq \alpha$, and $\gamma^{1}<\gamma^{0}$ if $\sum_{1 \leq l \leq j} \nu^{l}>j \alpha$. It follows from the definition of $\gamma^{0}$ and (4.19) that $A\left(\gamma^{0}, \alpha, j, \nu^{1}, \ldots, \nu^{j}\right) \circ v \sim 0$ for all $1 \leq j \leq J$ and $\nu^{1} \geq \alpha, \ldots, \nu^{j} \geq \alpha$ such that $\sum_{1 \leq l \leq j} \nu^{l}>j \alpha$. The claim is proved. The conclusion of the lemma follows by taking

$$
R_{\alpha}(X, x):=A\left(\gamma^{0}, \alpha, 0\right)+\sum_{j=1}^{J} A\left(\gamma^{0}, \alpha, j, \alpha, \ldots, \alpha\right) X^{j}
$$

since $\left.\left.R_{\alpha}\left(S^{\alpha} h(x), x\right)\right|_{x=v(y)} \sim S^{\gamma^{0}} P(h(x), x)\right|_{x=v(y)}$ and $S^{\gamma^{0}} P(h(x), x) \in \mathcal{J}$.

This completes the proof of Theorem 2.1.

\section{$\S 5$. Proof of Theorem 2.5}

The proof of Theorem 2.5 is parallel to that of Theorem 2.1, and we shall keep the notation from $\S 4$. We again use the notation $v^{k}(z, \xi), z \in \mathbb{C}^{n}$ and $\xi \in \mathbb{C}^{(k-1) n}$, for the $k$ th (formal) Segre mapping of $M$ at 0 . Let $H$ be the formal mapping given in the statement of Theorem 2.5. In what follows, $\mathcal{F}\left(M, M^{\prime}\right)$ will denote the set of all formal mappings $\left(\mathbb{C}^{N}, 0\right) \rightarrow\left(\mathbb{C}^{N^{\prime}}, 0\right)$ that send $M$ into $M^{\prime}$. Consider the following property, for $k \geq 0$ and $\alpha \in \mathbb{Z}_{+}^{N}$.

$(* *)_{k, \alpha}$ There exists $K(k, \alpha) \in \mathbb{Z}_{+}$such that for any $H^{\prime} \in \mathcal{F}\left(M, M^{\prime}\right)$ with

$$
\partial^{\beta} H(0)=\partial^{\beta} H^{\prime}(0), \forall|\beta| \leq K(k, \alpha),
$$

the following holds:

$$
\left(\partial^{\alpha} H\right) \circ v^{k} \sim\left(\partial^{\alpha} H^{\prime}\right) \circ v^{k} .
$$

Observe that $(* *)_{0, \alpha}$ holds with $K(0, \alpha)=|\alpha|$, since $v^{0}$ is the constant mapping into 0 . We shall prove that $(* *)_{k, \alpha}$ holds for all $k$ and $\alpha$ by induction on $k$ and $\alpha$. First assume $(* *)_{l, \beta}$ holds for $0 \leq l \leq k$ and all $\beta \in \mathbb{Z}_{+}^{N}$. We shall prove 
$(* *)_{k+1,0}$; i.e. we must show the existence of the integer $K(k+1,0)$ in $(* *)_{k+1,0}$. Let $P_{j}\left(X, Z, \zeta,\left(a_{\gamma}\right)_{|\gamma| \leq r}\right), j=1, \ldots, N^{\prime}$, be the polynomials in $X$ given by Theorem 3.1. Pick an integer $\tilde{K} \geq 0$ and consider the formal mappings $H^{\prime} \in \mathcal{F}\left(M, M^{\prime}\right)$ satisfying

$$
\partial^{\beta} H(0)=\partial^{\beta} H^{\prime}(0), \forall|\beta| \leq \tilde{K} .
$$

By a priori requiring $\tilde{K} \geq r$, where $r$ is the integer given by Theorem 3.1 , we may assume that any $H^{\prime} \in \mathcal{F}\left(M, M^{\prime}\right)$ that satisfies (5.3) also satisfies (3.3) with $H$ replaced by $H^{\prime}$. If we also take $\tilde{K} \geq K(k, \beta)$, for all $|\beta| \leq r$, then $(* *)_{k, \beta}$ implies that (5.2) holds for $|\beta| \leq r$. It follows from the above and Proposition 1.2.8 that $X=H_{j} \circ v^{k+1}$ and $X=H_{j}^{\prime} \circ v^{k+1}$ are both solutions of the equation

$$
P_{j}^{k+1}(X, z, \xi):=P_{j}\left(X, v^{k+1}(z, \xi), \bar{v}^{k}(\xi),\left(\partial^{\gamma} \bar{H}\left(\bar{v}^{k}(\xi)\right)-\partial^{\gamma} \bar{H}(0)\right)_{|\gamma| \leq r}\right) \sim 0 .
$$

Hence, if $m\left(P_{j}^{k+1}\right)$ is the integer given by Lemma 4.3 and we choose $K(k+1,0)=$ $\max \left(\tilde{K}, m\left(P_{j}^{k+1}\right)_{1 \leq j \leq N^{\prime}}\right)$, then the identity $H \circ v^{k+1} \sim H^{\prime} \circ v^{k+1}$ follows from Lemma 4.3. The property $(* *)_{k+1,0}$ is proved.

We now fix an integer $k$ and a multi-index $\alpha^{0}$. We complete the induction by assuming $(* *)_{l, \beta}$ for all pairs $l, \beta$ satisfying either $0 \leq l<k$ and $\beta \in \mathbb{Z}_{+}^{N}$ or $l=k$ and $\beta<\alpha^{0}$ in the lexicographic ordering of $\mathbb{Z}_{+}^{N}$. We shall prove $(* *)_{k, \alpha^{\prime}}$, where $\alpha^{\prime}$ is the multi-index immediately following $\alpha^{0}$ in the ordering of $\mathbb{Z}_{+}^{N}$. Again, consider those $H^{\prime} \in \mathcal{F}\left(M, M^{\prime}\right)$ that satisfy (5.3) with $\tilde{K} \geq r$. As above, the components $H_{j}^{\prime}$ of such an $H^{\prime}$ satisfy the same identities (3.3) as $H_{j}, j=1, \ldots, N^{\prime}$. By Lemma 4.9 , there exist formal series $R_{j \alpha^{\prime}}(X, Z, \zeta), j=1, \ldots, N^{\prime}$, in $\mathbb{C}[[Z, \zeta]][X]$ such that

$$
R_{j \alpha^{\prime}}\left(X, v^{k}(z, \xi), \bar{v}^{k-1}(\xi)\right) \nsim 0
$$

and

$$
R_{j \alpha^{\prime}}\left(\partial^{\alpha^{\prime}} H_{j}\left(v^{k}(z, \xi)\right), v^{k}(z, \xi), \bar{v}^{k-1}(\xi)\right) \sim 0 .
$$

Moreover, the coefficients of $R_{j \alpha^{\prime}}$, as a polynomial in $X$, are polynomials in $\partial^{\beta} H_{j}(Z)$ for $\beta<\alpha^{\prime}$, and $S^{\gamma} c^{j l}\left(Z, \zeta,\left(\partial^{\delta} \bar{H}_{j}(\zeta)-\partial^{\delta} \bar{H}_{j}(0)\right)_{|\delta| \leq r}\right), \gamma \leq N_{j} \alpha^{\prime}$, where $c^{j l}$ are given by (3.2). If $H^{\prime} \in \mathcal{F}\left(M, M^{\prime}\right)$ satisfies (5.3), then there is a corresponding formal series $R_{j \alpha^{\prime}}^{\prime}(X, Z, \zeta)$ for $H^{\prime}$ which is obtained from the same equation (5.4) with $H$ replaced by $H^{\prime}$. Hence, by Lemma 4.9 , if

$$
\begin{aligned}
& \left(\partial^{\beta} H\right) \circ v^{k} \sim\left(\partial^{\beta} H^{\prime}\right) \circ v^{k}, \beta<\alpha^{\prime}, \\
& \left(\partial^{\gamma} H\right) \circ v^{k-1} \sim\left(\partial^{\gamma} H^{\prime}\right) \circ v^{k-1},|\gamma|<r+\left|\alpha^{\prime}\right| \max \left(N_{j}\right)_{j=1, \ldots, N^{\prime}},
\end{aligned}
$$

then both $X=\partial^{\alpha^{\prime}} H \circ v^{k}$ and $X=\partial^{\alpha^{\prime}} H^{\prime} \circ v^{k}$ satisfy the same equation

$$
R_{j \alpha^{\prime}}^{k}(X, z, \xi):=R_{j \alpha^{\prime}}\left(X, v^{k}(z, \xi), \bar{v}^{k-1}(\xi)\right) \sim 0 .
$$

By the inductive hypothesis $(* *)_{l, \beta}$ for pairs $l, \beta$ as described above, (5.6) holds if $\tilde{K} \geq K(k, \beta), \beta<\alpha^{\prime}$, and $\tilde{K} \geq K(k-1, \gamma),|\gamma|<r+\left|\alpha^{\prime}\right| \max \left(N_{j}\right)_{j=1, \ldots, N^{\prime}}$. The property $(* *)_{k, \alpha^{\prime}}$ follows from Lemma 4.3 if we choose $K\left(k, \alpha^{\prime}\right)$ to be the maximum of $\tilde{K}$, as described above, and $m\left(R_{j \alpha^{\prime}}^{k}\right)$ for $j=1, \ldots, N^{\prime}$. This completes the induction, and proves that $(* *)_{k, \alpha}$ holds for all $k$ and $\alpha$.

To complete the proof of Theorem 2.5, recall that $M$ is of finite type at the origin and let $k_{1}$ be the integer obtained by applying Theorem 1.2.11 to $M$. If 
$H^{\prime} \in \mathcal{F}\left(M, M^{\prime}\right)$ satisfies (5.3) with $\tilde{K}=K\left(k_{1}, 0\right)$ then, by property $(* *)_{k_{1}, 0}$, we have

$$
H \circ v^{k_{1}} \sim H^{\prime} \circ v^{k_{1}} .
$$

Since $\operatorname{rk}\left(v^{k_{1}}\right)=N$ the components of the formal mapping $v^{k_{1}}$ cannot satisfy a nontrivial formal relation (see e.g. BER4, Proposition 5.3.5]). Hence, by (5.8) we must have $H_{j}-H_{j}^{\prime} \sim 0$. This completes the proof of Theorem 2.5.

\section{§6. Proofs of Theorems 3, 4, AND 5}

For the proofs of Theorems 3, 4, and 5, we shall need the following two lemmas.

Lemma 6.1. Let $M$ and $M^{\prime}$ be formal generic submanifolds of the same dimension through the origin in $\mathbb{C}^{N}$. Assume that $H:\left(\mathbb{C}^{N}, 0\right) \rightarrow\left(\mathbb{C}^{N}, 0\right)$ is a finite formal mapping sending $M$ into $M^{\prime}$. Then the Segre homomorphism $\Phi_{H}$ is injective.

Proof. Since $M$ and $M^{\prime}$ are generic and of the same dimension, we have $n=n^{\prime}$ and $d=d^{\prime}$. Let $Z=(z, w)$ and $Z^{\prime}=\left(z^{\prime}, w^{\prime}\right)$ be normal coordinates for $M$ and $M^{\prime}$, respectively, and write $H=(F, G)$ as in $\S 1.2$. Recall that injectivity of the Segre homomorphism $\Phi_{H}$ is equivalent to the fact that there is no nontrivial formal power series $h \in \mathbb{C}\left[\left[z^{\prime}\right]\right]$ such that $h(F(z, 0)) \sim 0$. We first claim that the formal mapping $z \mapsto F(z, 0)$ is again finite. To see this, consider the homomorphism $\psi: \mathbb{C}[[z, w]] \rightarrow \mathbb{C}[[z]]$ defined by $\psi(f)(z)=f(z, 0)$. Observe that $\psi$ is surjective and maps the ideal $\mathcal{I}(F(z, w), G(z, w))$ into $\mathcal{I}(F(z, 0))$ since $G(z, 0) \sim 0$ by normality of the coordinates. Hence, $\psi$ induces a surjective homomorphism

$$
\tilde{\psi}: \mathbb{C}[[z, w]] / \mathcal{I}(F(z, w), G(z, w)) \rightarrow \mathbb{C}[[z]] / \mathcal{I}(F(z, 0)) .
$$

Since the vector space on the left-hand side of (6.2) is finite dimensional and $\tilde{\psi}$ is surjective, we conclude that the vector space on the right-hand side of (6.2) is also finite dimensional. This proves the claim above. To complete the proof of the lemma, we must show that any finite formal mapping $K:\left(\mathbb{C}^{k}, 0\right) \rightarrow\left(\mathbb{C}^{k}, 0\right)$ induces an injective ring homomorphism $\eta_{K}: \mathbb{C}[[x]] \rightarrow \mathbb{C}[[x]$. This can be proved by showing that the Jacobian determinant $\operatorname{det}(\partial K / \partial x)$ cannot vanish identically (see e.g. [BER4 Theorem 5.1.37]) and that the latter implies that there is no nontrivial $h \in \mathbb{C}[[x]]$ such that $h(K(x)) \sim 0$ (see [BER4 Proposition 5.3.5]).

Lemma 6.3. Let $M$ be a formal generic submanifold through the origin in $\mathbb{C}^{N}$. If $M$ does not contain a nontrivial formal variety through 0 , then $M$ is essentially finite at 0 . Moreover, if $M$ is real-analytic and does not contain a nontrivial holomorphic variety through 0 , then $M$ is essentially finite at 0.

Proof. Let $d$ be the codimension of $M$. If $n:=N-d=0$, then there is nothing to prove since every such generic submanifold is essentially finite at 0 . (In this case, there are no CR vector fields tangent to $M$ and the condition for essential finiteness is void.) Assume $n \geq 1$ and that $M$ does not contain any nontrivial formal curve through 0 . Let $Z=(z, w)$ be normal coordinates for $M$ as in $\S 1.2$. Thus, $M$ is given by (1.2.1). To check that $M$ is essentially finite at 0 we use the expansion (1.2.5) and must show that the ideal generated by $\bar{q}_{j \alpha}(z, 0), j=1, \ldots, d$ and $\alpha \in \mathbb{Z}_{+}^{n}$, in $\mathbb{C}[[z]]$ has finite codimension. If not, then by Lemma 3.32 there would exist a nontrivial formal curve $\mu:(\mathbb{C}, 0) \rightarrow\left(\mathbb{C}^{n}, 0\right)$ such that

$$
\bar{q}_{j \alpha}(\mu(s), 0) \sim 0, \quad j=1, \ldots, d, \alpha \in \mathbb{Z}_{+}^{n} .
$$


This would contradict the assumption that $M$ does not contain a nontrivial formal curve through 0 , since, as the reader can easily verify, it would follow that $s \mapsto$ $(\mu(s), 0)$ is a nontrivial formal curve through 0 contained in $M$. This proves the first part of the lemma. The proof of the last statement in Lemma 6.3, which is more standard, is completely analogous and uses the Nullstellensatz instead of Lemma 3.32. (See e.g. [BER4, Chapter XI].)

We now give the proofs of the results in the introduction.

Proof of Theorems 3 and 5. By Lemmas 6.1 and 6.3, Theorem 3 is an immediate consequence of Theorem 2.1. Similarly, Theorem 5 follows by applying Theorem 2.5 to the formal submanifolds and the formal mapping associated to $M, M^{\prime}$ and $H$, respectively.

Proof of Theorem 4. Assume that $H:\left(\mathbb{C}^{N}, 0\right) \rightarrow\left(\mathbb{C}^{N}, 0\right)$ is a formal invertible mapping sending $M$ into $M^{\prime}$. Since the Segre homomorphism of $H$ is injective, Theorem 4 is a consequence of Theorem 2.1 provided that we can show that $M^{\prime}$ is essentially finite at 0 . Indeed, since $M$ is essentially finite (by Lemma 6.3) and the notion of essential finiteness is invariant under formal invertible mappings, it follows that $M^{\prime}$ is also essentially finite at 0 .

\section{$\S 7$. Proofs of Theorems $1,2.2$, And 2.4}

For the proof of Theorem 2.2, we shall use Theorem 2.1 and the following result.

Proposition 7.1. Let $M$ and $M^{\prime}$ be formal hypersurfaces through the origin in $\mathbb{C}^{N}$. Assume that $M$ is essentially finite at 0 and $M^{\prime}$ does not contain any nontrivial formal variety through 0 . If $H:\left(\mathbb{C}^{N}, 0\right) \rightarrow\left(\mathbb{C}^{N}, 0\right)$ is a formal mapping sending $M$ into $M^{\prime}$, then either $H(Z) \sim 0$ or the Segre homomorphism $\Phi_{H}$ is injective.

Proof. Let $Z=(z, w)$ and $Z^{\prime}=\left(z^{\prime}, w^{\prime}\right)$ be normal coordinates for $M$ and $M^{\prime}$, respectively, as in $\S 1.2$. Thus, $M$ is given by (1.2.1) and similarly for $M^{\prime}$ with $Q$ replaced by $Q^{\prime}$. We write $H=(F, G)$. We shall prove that if $H \nsim 0$, then the mapping $z \mapsto F(z, 0)$ is finite, which implies injectivity of $\Phi_{H}$ as noted in the proof of Lemma 6.1. The following is proved in BR (Theorem 2). Suppose that $M$ and $M^{\prime}$ are formal hypersurfaces through 0 in $\mathbb{C}^{N}$ with $M$ essentially finite at 0 . If $H:\left(\mathbb{C}^{N}, 0\right) \rightarrow\left(\mathbb{C}^{N}, 0\right)$ is a formal mapping sending $M$ into $M^{\prime}$ and if the transversal component $G \nsim 0$ (where $M$ and $M^{\prime}$ are written in normal coordinates as above and $H=(F, G)$ ), then the formal mapping $z \mapsto F(z, 0)$ is finite. Hence, to prove Proposition 7.1 it suffices to show that $G \sim 0$ implies $F \sim 0$.

Thus, assume that $G \sim 0$. The fact that $H$ sends $M$ into $M^{\prime}$ is then expressed by

$$
Q^{\prime}(F(z, w), \bar{F}(\chi, \tau), 0) \in \mathcal{I}(M) .
$$

We claim that (7.2) implies

$$
Q^{\prime}(F(z, w), \bar{F}(\chi, \tau), 0) \sim 0 .
$$

To see this, observe that by linear algebra there exists a nontrivial formal holomorphic vector field

$$
X=\sum_{j=1}^{n} a_{j}(z, w) \frac{\partial}{\partial z_{j}}+b(z, w) \frac{\partial}{\partial w}, \quad a_{j}, b \in \mathbb{C}[[z, w]]
$$


such that

$$
X F_{j}(z, w) \sim 0, \quad j=1, \ldots, n .
$$

Since $M$ is essentially finite at $0, X$ cannot be tangent to $\mathcal{I}(M)$ (see BER4, Theorem 11.8.13]; the property of having no nontrivial formal holomorphic vector fields tangent is referred to as holomorphic nondegeneracy of $M$ at 0$)$. By (7.2), if (7.3) does not hold, then there exists $a \in \mathbb{C}[[z, w, \chi, \tau]]$ and an integer $k \geq 1$ such that

$$
Q^{\prime}(F(z, w), \bar{F}(\chi, \tau), 0) \sim a(z, w, \chi, \tau) \rho(z, w, \chi, \tau)^{k},
$$

where $\rho$ is some generator of $\mathcal{I}(M)$ (e.g. $\rho(z, w, \chi, \tau):=w-Q(z, \chi, \tau)$ ), and $a \notin$ $\mathcal{I}(M)$. If we apply $X$ to (7.6), use (7.5), and divide by $\rho^{k-1}$, then we deduce

$$
0 \sim(X a) \rho+k a(X \rho) .
$$

We conclude that $a(X \rho) \in \mathcal{I}(M)$ and, hence since $\mathcal{I}(M)$ is prime, either $a \in \mathcal{I}(M)$ or $X \rho \in \mathcal{I}(M)$. Both cases are impossible and consequently the claim (7.3) is proved. If $F(z, w) \nsim 0$, then there exists a nontrivial formal curve $s \mapsto(z(s), w(s))$, vanishing at $s=0$, such that the formal curve $s \mapsto F(z(s), w(s))$ is nontrivial. Since the formal curve $s \mapsto(F(z(s), w(s)), 0)$ is contained in $M^{\prime}$ (which is assumed not to contain a nontrivial formal curve), by (7.3), we conclude that $F(z, w)$ must be identically 0. This completes the proof of Proposition 7.1.

Proof of Theorem 2.2. Since the hypersurface $M$ is essentially finite at 0 , it is also of finite type at 0 (see e.g. [BER4, Proposition 9.4.16]). By the assumption on $M^{\prime}$ and Lemma $6.3, M^{\prime}$ is essentially finite at 0 . Moreover, we claim that $M^{\prime}$ does not contain a nontrivial formal curve through 0 . Indeed, if the real-analytic hypersurface $M^{\prime}$ were to contain a nontrivial formal curve through 0 , then $M^{\prime}$ would be of infinite type at 0 in the sense of D'Angelo. Hence, $M^{\prime}$ would contain a nontrivial holomorphic subvariety (see e.g. [DA, Section 3.3.3]), which would contradict the assumption on $M^{\prime}$. (One could also apply directly the theorem of Milman [Mi] which states that if a real-analytic variety contains a formal variety, then it also contains a holomorphic one of the same dimension.) The proof of Theorem 2.2 now follows from Theorem 2.1 and Proposition 7.1.

Proof of Theorem 1. Theorem 1 follows from Theorem 2.2 by using Lemma 6.3.

Proof of Theorem 2.4. To prove Theorem 2.4, we denote by $H(Z)$ the formal mapping $\left(\mathbb{C}^{N}, 0\right) \rightarrow\left(\mathbb{C}^{N}, 0\right)$ given by the Taylor series of $h$ at 0 (see [BER4, Proposition 1.7.14]). By assumption, $H(Z) \nsim 0$. As explained in the proof of Theorem 2.2 above, $M^{\prime}$ does not contain a nontrivial formal curve through 0 . We take normal coordinates for $M$ and $M^{\prime}$ and write $H=(F, G)$ as in $\S 1.2$. Proposition 7.1 implies that the Segre homomorphism $\Phi_{H}$ is injective. Indeed, the proof of Proposition 7.1 in fact shows that the mapping $z \mapsto F(z, 0)$ is finite. It follows (see e.g. BER4. Theorem 5.1.37]) that $H$ is not totally degenerate, i.e.

$$
\operatorname{det}\left(\partial F_{i} / \partial z_{j}\right)_{1 \leq i, j \leq n}(z, 0) \nsim 0 \text {. }
$$

Theorem 2.4 now follows from the reflection principle in $\mathrm{BR}$, Theorem 6] (see also [BER4 Theorem 9.6.1]), since $M^{\prime}$ is essentially finite at 0 by Lemma 6.3.

Closing remark. During the completion of this work, the authors became aware of the preprint "Convergence of formal biholomorphisms between minimal holomorphically nondegenerate real analytic CR manifolds" (e-print : http://xxx.lanl.gov/ 
abs/math.CV/9901027; 1999) by J. Merker in which convergence of formal invertible mappings between holomorphically nondegenerate generic submanifolds of finite type is claimed. However, the proof in that preprint has serious flaws. In particular, the proof is based on Proposition 2.4.6 (in that preprint) which is an incorrect characterization of holomorphic nondegeneracy for minimal generic submanifolds. The real algebraic hypersurface in $\mathbb{C}^{3}$ defined by

$$
\operatorname{Im} w=\frac{\left|z_{1}\right|^{2}\left|1+z_{1} \bar{z}_{2}\right|^{2}}{1+\operatorname{Re}\left(z_{1} \bar{z}_{2}\right)}-\operatorname{Re} w \frac{\operatorname{Im}\left(z_{1} \bar{z}_{2}\right)}{1+\operatorname{Re}\left(z_{1} \bar{z}_{2}\right)}
$$

gives a counterexample to that proposition.

\section{ACKNOWLEDGEMENTS}

The authors would like to thank J. D'Angelo, X. Gong, and X. Huang for helpful

bibliographical information, and also C. Huneke for his help with the proof of Lemma 3.32 .

\section{REFERENCES}

[A] M. Artin, On the solution of analytic equations, Invent. Math. 5 (1969), 277-291. MR 38:344

[AM] M. F. Atiyah and I. G. Macdonald, Introduction to Commutative Algebra, AddisonWesley, Reading, MA, 1969. MR 39:4129

[BER1] M. S. Baouendi, P. Ebenfelt, and L. P. Rothschild, Algebraicity of holomorphic mappings between real algebraic sets in $\mathbb{C}^{n}$, Acta Math. 177 (1996), 225-273. MR 99b:32030

[BER2] — CR automorphisms of real analytic CR manifolds in complex space, Comm. Anal. Geom. 6 (1998), 291-315. [MR 99i:32024

[BER3] _ Parametrization of local biholomorphisms of real analytic hypersurfaces, Asian J. Math. 1 (1997), 1-16. MR 99b:32022

[BER4] , Real Submanifolds in Complex Space and Their Mappings, Princeton Math. Ser. 47, Princeton Univ. Press, Princeton, NJ, 1999. MR 2000b:32066

[BER5] _ Rational dependence of smooth and analytic CR mappings on their jets, Math. Ann. 315 (1999), 205-249. CMP 2000:04

[BR] M. S. Baouendi and L. P. Rothschild, Geometric properties of mappings between hypersurfaces in complex space, J. Differential Geom. 31 (1990), 473-499. MR 91g:32032

[B] V. K. Beloshapka, A uniqueness theorem for automorphisms of a nondegenerate surface in complex space, Math. Notes 47 (1990), 239-242. MR 91j:32019

[BK] E. Brieskorn and H. Knörrer, Plane Algebraic Curves (Translated from the German by John Stillwell), Birkhäuser Verlag, Basel-Boston, Mass., 1986. MR 88a:14001

[C] E. Cartan, Sur la géométrie pseudo-conforme des hypersurfaces de deux variables complexes, I, Ann. Math. Pura Appl. 11 (1932), 17-90 (or Oeuvres II, 1231-1304).

[DA] J. D'Angelo, Several Complex Variables and the Geometry of Hypersurfaces, Studies in Advanced Math., CRC Press, Boca Raton, 1993. MR 94i:32022

[CM] S. S. Chern and J. K. Moser, Real hypersurfaces in complex manifolds, Acta Math. 133 (1974), 219-271. MR 54:13112

[Go1] X. Gong, Divergence of the normalization of real-analytic glancing hypersurfaces, Comm. Partial Differential Eq. 19 (1994), 643-654. MR 95f:58079

[Go2] - Divergence of the normalization for real Lagrangian surfaces near complex tangents, Pacific J. Math. 176 (1996), 311-324. MR 98e:32028

[Gu] R. C. Gunning, Introduction to Holomorphic Functions of Several Variables., Vols. I, II, and III, The Wadsworth \& Brooks/Cole Mathematics Series, Pacific Grove, CA, 1990. MR 92b:32001a MR 92b:32001b MR 92b:32001c

[Ha] R. Hartshorne, Algebraic Geometry, Springer-Verlag, Berlin, 1993. MR 82c:14003 (review of 1979 edition)

$[\mathrm{Hu}] \mathrm{X}$. Huang, Schwarz reflection principle in complex spaces of dimension two, Comm. Partial Differential Eq. 21 (1996), 1781-1828. MR 97m:32043 
[Mel] R. B. Melrose, Equivalence of glancing hypersurfaces, Invent. Math. 37 (1976), 165-191. MR 55:9173

[Mi] P. Milman, Complex analytic and formal solutions of real analytic equations in $\mathbb{C}^{n}$, Math. Ann. 233 (1978), 1-7. |MR 58:6322

$[\mathrm{MW}]$ J. K. Moser and S. M. Webster, Normal forms for real surfaces in $\mathbb{C}^{2}$ near complex tangents and hyperbolic surface transformations, Acta Math. 150 (1983), 255-296. MR 85c:32034

[O] T. Oshima, On analytic equivalence of glancing hypersurfaces, Sci. Papers College Gen. Ed. Univ. Tokyo 28 (1978), 51-57. MR 58:13231

[T] N. Tanaka, On the pseudo-conformal geometry of hypersurfaces of the space of $n$ complex variables, J. Math. Soc. Japan 14 (1962), 397-429. MR 26:3086

[TH] A. Tumanov and G. M. Henkin, Local characterization of holomorphic automorphisms of Siegel domains, Funktsional. Anal. i Prilozhen 17, 49-61; English transl. in. Functional Anal. Appl. 17 (1983). MR 86a:32063

[VW] B. L. Van der Waerden, Modern Algebra, Eighth Edition, Springer-Verlag, New York, NY, 1971. MR 10:587b (review of 1949 edition)

[W] S. M. Webster, Holomorphic symplectic normalization of a real function, Ann. Scuola Norm. Pisa 19 (1992), 69-86. MR 94d:32024

[Z] D. Zaitsev, Germs of local automorphisms of real-analytic CR structures and analytic dependence on $k$-jets, Math. Research Lett. 4 (1997), 823-842. MR 99a:32007.

[ZS] O. Zariski and P. Samuel, Commutative Algebra, Vols. I and II, Springer-Verlag, New York, NY, 1958, 1960. MR 19:833e] MR 22:11006

Department of Mathematics, 0112, University of California at San Diego, La Jolla, CALIFORNIA 92093-0112

E-mail address: sbaouendi@ucsd.edu

Department of Mathematics, Royal Institute of Technology, S-100 44 Stockholm, SWEDEN

E-mail address: ebenfelt@math.kth.se

Department of Mathematics, 0112, University of California at San Diego, La Jolla, CALIFORNia 92093-0112

E-mail address: lrothschild@ucsd.edu 